\title{
REVISITING SPITZER TRANSIT OBSERVATIONS WITH INDEPENDENT COMPONENT ANALYSIS: NEW RESULTS FOR THE GJ 436 SYSTEM
}

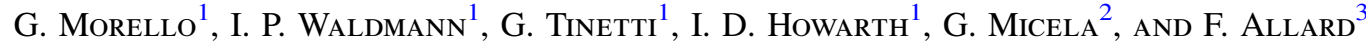 \\ ${ }^{1}$ Department of Physics \& Astronomy, University College London, Gower Street, WC1E6BT, UK; giuseppe.morello.11@ucl.ac.uk \\ ${ }^{2}$ INAF-Osservatorio Astronomico di Palermo, Piazza del Parlamento 1, I-90134, Italy \\ ${ }^{3}$ Centre de Recherche Astrophysique de Lyon-École Normale Supérieure de Lyon, 46 Allée d'Italie, 69364 Lyon Cedex 07, France \\ Received 2014 September 27; accepted 2015 January 22; published 2015 April 1
}

\begin{abstract}
We analyzed four Spitzer/IRAC observations at 3.6 and $4.5 \mu \mathrm{m}$ of the primary transit of the exoplanet GJ 436b, by using blind source separation techniques. These observations are important for investigating the atmospheric composition of the planet GJ 436b. Previous analyses claimed strong inter-epoch variations of the transit parameters due to stellar variability, casting doubts on the possibility of conclusively extracting an atmospheric signal. Those analyses also reported discrepant results, hence the necessity of this reanalysis. The method we used has been proposed in Morello et al. to analyze $3.6 \mu \mathrm{m}$ transit light curves of the hot Jupiter HD 189733b. It performes an Independent Component Analysis on a set of pixel light curves, i.e., time series read by individual pixels, from the same photometric observation. Our method only assumes the independence of instrumental and astrophysical signals, and therefore guarantees a higher degree of objectivity compared to parametric detrending techniques published in the literature. The data sets we analyzed in this paper represent a more challenging test than the previous ones. Contrary to previous results reported in the literature, our results (1) do not support any detectable inter-epoch variations of orbital and stellar parameters, (2) are photometrically stable at the level $\sim 10^{-4}$ in the IR, and (3) the transit depth measurements at the two wavelengths are consistent within $1 \sigma$. We also (4) detect a possible transit duration variation of $\sim 80 \mathrm{~s}(2 \sigma$ significance level) that has not been pointed out in the literature, and (5) confirm no transit timing variations $\gtrsim 30 \mathrm{~s}$.
\end{abstract}

Key words: methods: data analysis - planets and satellites: atmospheres - planets and satellites: individual (GJ 436b) - techniques: photometric

\section{INTRODUCTION}

Transit spectroscopy and differential photometry are largely used to investigate the composition and structure of exoplanetary atmospheres. The large majority of transiting exoplanets are "hot Jupiters," i.e., planets similar in size to Jupiter orbiting very closely to their host star (semimajor axis $\sim 0.01-0.5 \mathrm{AU}$ ). Their typical surface temperatures are $\gtrsim 1000 \mathrm{~K}$.

GJ 436b is a Neptune-sized planet orbiting around an M dwarf with radius $\sim 0.46 R_{\odot}$ at a distance $\sim 0.03 \mathrm{AU}$. This planet is interesting for several reasons. It is one of the smallest (radius $\sim 4.3 R_{\oplus}$ ) and coolest $(\sim 700 \mathrm{~K})$ exoplanets for which optical-to-IR spectra have been measured (Demory et al. 2007; Gillon et al. 2007; Alonso et al. 2008; Coughlin et al. 2008; Cáceres et al. 2009; Deming et al. 2009; Pont et al. 2009; Ballard et al. 2010; Stevenson et al. 2010; Beaulieu et al. 2011; Knutson et al. 2011, 2014). The primary transit depth is $\sim 0.7 \%$. Another peculiarity of GJ $436 \mathrm{~b}$ is its high orbital eccentricity $(e \sim 0.16)$, inferred from radial velocity measurements (Maness et al. 2007) and from secondary eclipse phasing (Deming et al. 2009). Both the physical and dynamical properties of GJ 436b are debated in the literature.

Maness et al. (2007) and Demory et al. (2007) investigated the origin of the high orbital eccentricity of GJ 436b, concluding that the circularization timescale $\left(\sim 10^{8} \mathrm{yr}\right)$ is significantly smaller than the age of the system $\left(\gtrsim 6 \times 10^{9}\right)$. Maness et al. (2007) also found a long trend in radial velocity measurements. They suggested the presence of an external perturber on a wider orbit to explain both the high eccentricity of GJ $436 \mathrm{~b}$ and the long trend in radial velocity measurements. Ribas et al. (2008) hypothesized a Super-Earth on a close orbit to explain those evidences, but later retracted. Transit timing variations (TTVs) reported by Alonso et al. (2008) and Cáceres et al. (2009) do not support any evidence of external perturbers. Stevenson et al. (2012) claimed the possible detection of two nearby sub-Earth-sized exoplanets transiting in GJ 436 system; according to the authors, the dynamic of the proposed system is consistent with the current non-TTVdetections.

Based on multiwavelength infrared eclipse measurements, Stevenson et al. (2010) proposed a high $\mathrm{CO}$-to- $\mathrm{CH}_{4}$ ratio compared to thermochemical equilibrium models for hydrogendominated atmospheres. Their atmospheric model includes disequilibrium processes, such as vertical mixing and polymerization of methane to explain the observed deficiency of $\mathrm{CH}_{4}$. Beaulieu et al. (2011) suggested strong $\mathrm{CH}_{4}$ absorption at 3.6, 4.5, and $8.0 \mu \mathrm{m}$ Spitzer/IRAC passbands from primary transit observations, and their reanalysis of secondary eclipse data is consistent with this detection. Knutson et al. (2011) measured significant time variations of the transit depths at the same wavelengths, which strongly affects the inferred transmission spectrum. They attributed such variations to the stellar activity and found that different results are obtainable depending on the observations considered. By rejecting those observations that they believe to be most strongly affected by stellar activity, their final results support $\mathrm{CO}$ as the dominant carbon molecule, with very little, if any, $\mathrm{CH}_{4}$. More recent Hubble/WFC3 observations in the $1.2-1.6 \mu \mathrm{m}$ wavelength interval, analyzed by Knutson et al. (2014), indicate a featureless transmission spectrum, which is consistent with a relatively hydrogen-poor atmosphere with a high cloud or haze layer. 
Table 1

Spitzer Observations of Primary Transits of GJ 436b

\begin{tabular}{lcccc}
\hline \hline Obs. Number & Detector & Wavelength $(\mu \mathrm{m})$ & UT Date & Orbit Number \\
\hline $1 \mathrm{a}$ & IRAC, ch1 & 3.6 & 2009 Jan 9 & 234 \\
$1 \mathrm{~b}$ & IRAC, ch1 & 3.6 & 2009 Jan 28 & 241 \\
$2 \mathrm{a}$ & IRAC, ch2 & 4.5 & 2009 Jan 17 & 237 \\
$2 \mathrm{~b}$ & IRAC, ch2 & 4.5 & 2009 Jan 31 & 242 \\
\hline
\end{tabular}

In this paper we reanalyze four transit light curves obtained with Spitzer/IRAC at 3.6 and $4.5 \mu \mathrm{m}$ passbands (channels 1 and 2 of IRAC). We adopt a non-parametric data detrending technique, based on Independent Component Analysis (ICA) applied to single pixel light curves, to ensure a higher degree of objectivity. This method has been proven to give robust results when applied to the transits of the hot Jupiter HD 189733b observed with IRAC at $3.6 \mu \mathrm{m}$ (Morello et al. 2014). We further test the performance of this detrending technique with the more challenging data sets of the Neptune-sized planet GJ $436 \mathrm{~b}$, for which the transit depth is comparable with the amplitude of the instrumental pixel-phase signal, and the transit duration is very similar to the period of that signal. Additionally, we discuss the stellar and orbital stability of the GJ 436 system, the repeatability of transit measurements (potentially affected by stellar, planet, and instrument variability), and the atmospheric contribution. We also discuss the reliability of our results in light of other observations reported in the literature, in particular Beaulieu et al. (2011), Knutson et al. $(2011,2014)$.

\section{DATA ANALYSIS}

\subsection{Observations}

We analyze four photometric observations of GJ 436b, which are part of the Spitzer program ID 50051. They include two 3.6 and two $4.5 \mu \mathrm{m}$ primary transits, as detailed in Table 1. Each observation consists of 1,829 exposures using IRAC's sub-array mode, taken over $4.3 \mathrm{hr}$ : $0.8 \mathrm{hr}$ on the primary transit of the planet, the remaining $3.5 \mathrm{hr}$ before and after transit. The interval between consecutive exposures is $8.4 \mathrm{~s}$. Each exposure includes 64 consecutive frames integrated over $0.1 \mathrm{~s}$. We replaced the single frames of each exposure with their averages to reduce the random scatter and the computational time. ${ }^{4}$ During an observation, the centroid of the star GJ 436 is stable to within one pixel.

\subsection{Detrending Method, Light Curve Fitting, and Error Bars}

Here we outline the main steps of the analysis: data detrending, light-curve fitting, and estimating parameter error bars. Further details are reported in Morello et al. (2014).

To detrend the transit signals from single observations, we performed an ICA decomposition over selected pixel light curves, i.e., time series from individual pixels. We considered $5 \times 5$ arrays of pixels with the stellar centroids at their centers. In this way, we obtain a set of maximally independent components: one of them is the transit signal, others may be instrumental systematics and/or astrophysical signals. Observed light curves are linear combinations of these

\footnotetext{
4 Computational time is dominated by the time for transit fitting, which strongly depends on the algorithms (and settings) used. In our case, transit fitting time was several hours, and it scales as $\mathcal{O}(d N)$, being $d$ the number of free transit parameters, and $N$ the data points.
}

independent components, and the coefficients of the linear combinations can be calculated by fitting the out-of-transit parts. To estimate the transit signal in a robust way, the fit is performed on the out-of-transit of the relevant integral lightcurve, i.e., the sum of the pixel light curves from the array used, including all the non-transit components, plus a constant term. The detrended transit signal is obtained by subtracting all the non-transit components, properly scaled by their fitting coefficients, from the integral light-curve. It is renormalized by the mean value on the out-of-transit, so that the out-oftransit level is unity.

After the extractions of the detrended and normalized transit time series, we modeled them by using the Mandel \& Agol (2002) analytical formulae. We originally assumed the orbital period, $P$, and the epoch of the first transit, $E_{t r}$, reported by Cáceres et al. (2009); the eccentricity, $e$, and the argument of periastron, $\omega$, reported by Maness et al. (2007). They are consistent with those reported by previous papers (Butler et al. 2004; Gillon et al. 2007; Deming et al. 2009), and more accurate. We tested two different sets of quadratic limb darkening coefficients for the star (Howarth 2011b), $\gamma_{1}$ and $\gamma_{2}$, derived by an Atlas (Kurucz 1970; Howarth 2011a) and a Phoenix (Allard \& Hauschildt 1995; Allard et al. 2001) model (see Section 2.3.1). With these settings, we estimated the planet-to-star radii ratio, $p=\frac{r_{p}}{R_{s}}$, the orbital semimajor axis in units of stellar radii, $a_{0}=\frac{a}{R_{s}}$, and inclination, $i$. First estimates were obtained through a Nelder-Mead optimization algorithm (Lagarias et al. 1998); they were used as optimal starting points for an Adaptive Metropolis algorithm with delayed rejection (Haario et al. 2006), generating chains of 20,000 values. Updated best estimates and (partial) error bars of the parameters, $\sigma_{\mathrm{par}, 0}$, are the means and standard deviations of the relevant (Gaussian distributed) sampled chains, respectively. The final parameter error bars are:

$$
\sigma_{\mathrm{par}}=\sigma_{\mathrm{par}, 0} \sqrt{\frac{\sigma_{0}^{2}+\sigma_{\mathrm{ICA}}^{2}}{\sigma_{0}^{2}}}
$$

$\sigma_{0}^{2}$ is the sampled likelihood variance, approximately equal to the variance of the residuals for the best transit model; $\sigma_{\text {ICA }}^{2}$ is a term estimating the uncertainty associated with the ICA extraction (see Appendix A.3 for further details).

For completeness, and for comparison with the literature, we also calculated the transit depth, $p^{2}$, the impact parameter, $b$, and the transit duration, $T$, where (see Ford et al. 2008):

$$
\begin{gathered}
b=a_{0} \cos i \frac{1-e^{2}}{1+e \sin \omega} \\
T=\frac{P \sqrt{1-b^{2}}}{\pi a_{0}} \frac{\sqrt{1-e^{2}}}{1+e \sin \omega} .
\end{gathered}
$$



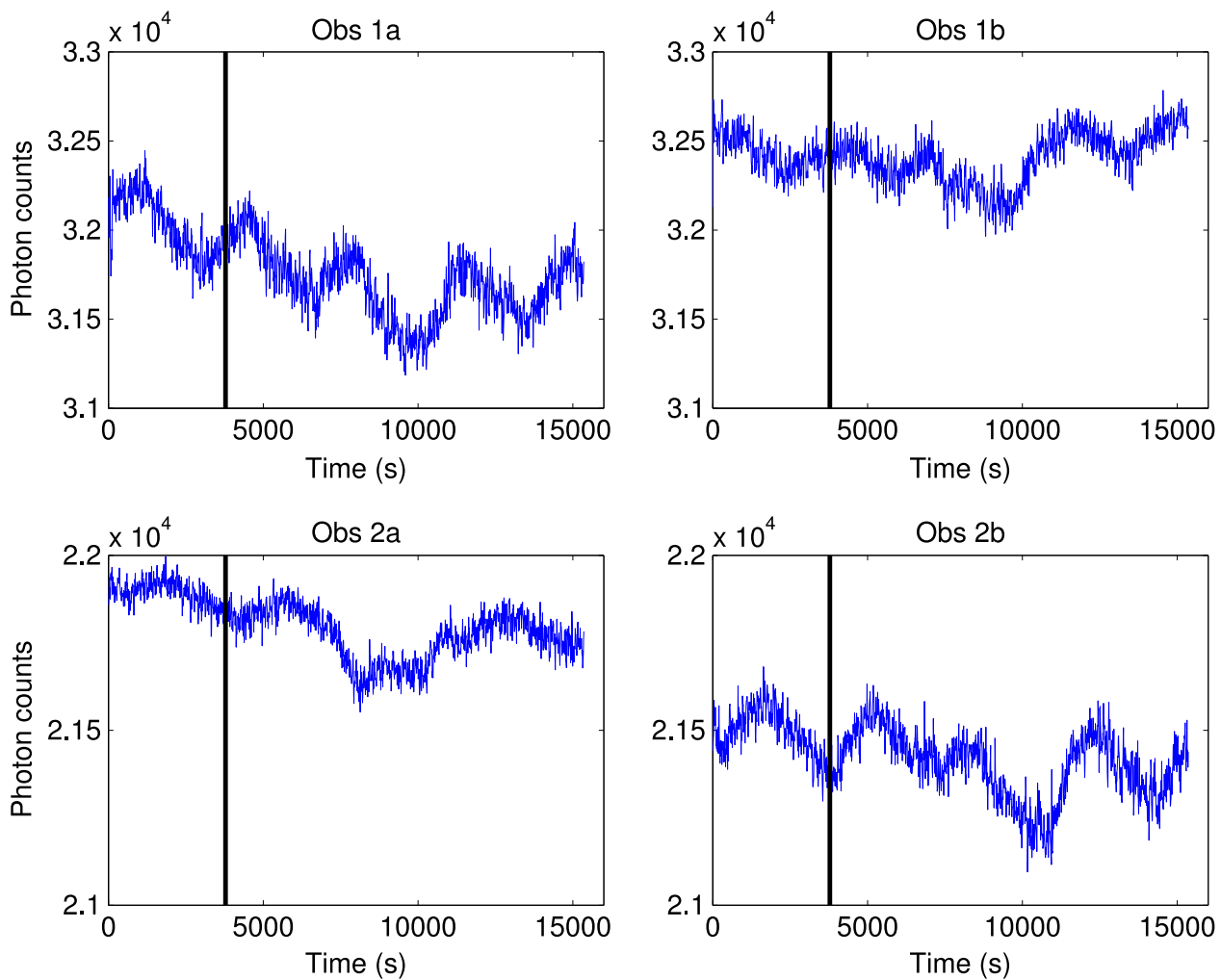

Figure 1. Raw integral light curves of the four primary transit observations. Data points on the left of the black vertical lines have been discarded for the analysis. Note that the transit depth is comparable with the amplitude of systematics.

Table 2

Quadratic Limb Darkening Coefficients Computed by Atlas and Phoenix Stellar Models for IRAC 3.6 and $4.5 \mu \mathrm{m}$ Bands

\begin{tabular}{lcc}
\hline \hline Atlas & $3.6 \mu \mathrm{m}$ & $4.5 \mu \mathrm{m}$ \\
\hline$\gamma_{1}$ & $5.489 \times 10^{-2}$ & $1.331 \times 10^{-2}$ \\
$\gamma_{2}$ & $3.0653 \times 10^{-1}$ & $2.8396 \times 10^{-1}$ \\
\hline Phoenix & $3.6 \mu \mathrm{m}$ & $4.5 \mu \mathrm{m}$ \\
\hline$\gamma_{1}$ & $3.87 \times 10^{-3}$ & $3.27 \times 10^{-3}$ \\
$\gamma_{2}$ & $2.3615 \times 10^{-1}$ & $1.8193 \times 10^{-1}$ \\
\hline
\end{tabular}

For a more thorough analysis, we performed other fits with different choices of the free parameters, introducing a phaseshift parameter to consider possible timing error/variations, and simultaneous fits on more than one multiple light curves with some common free parameters.

\subsection{Application to Observations}

Figure 1 reports the raw "integral light curves" observed and Figure 2 reports the corresponding central pixel light curves. The main systematic effect for IRAC channels 1 and 2 observations is an almost regular undulation with period $\sim 3000 \mathrm{~s}$, so-called pixel-phase effect, because it depends on the relative position of the source centroid with respect to a pixel center (Fazio et al. 2004; Morales-Caldéron et al. 2006). This effect is particularly difficult to detrend from these data sets because its timescale is similar to the transit duration, and its amplitude is comparable to the transit depth. Recently, a time dependence of the pixel-phase effect has been suggested (Stevenson et al. 2010; Beaulieu et al. 2011).
If considering the whole data sets for detrending, our ICA algorithm is able to remove most of the non-flatness on the outof-transits, and visibly improve the in-transit shapes, but some visible issues remain (see Figure 16). We noted that results improve significantly if a number of data points from the beginning of each observation are rejected. This is discussed on a statistical basis in Section 3.1. A possible explanation is that the first data points contain a long-tail variation until the stabilization of the instruments (Fazio et al. 2004, see also Appendix A.1), but this is not a crucial point for the data analysis. In the rest of this paper we discuss the results obtained after rejecting the first 450 exposures from each observation, corresponding to $\sim 3780 \mathrm{~s}$, for which the ICA performances are optimal. It is worth pointing out that different choices (including no data rejections) give consistent results (within $1 \sigma)$, with larger or similar error bars.

\subsubsection{Limb Darkening Coefficients}

Table 2 reports the quadratic limb darkening coefficients used at 3.6 and $4.5 \mu \mathrm{m}$ IRAC passbands. Both the Atlas and Phoenix models are computed with $T_{\text {eff }}=3680 \mathrm{~K}$, $\log g=4.78$ (Torres 2009), and solar abundances (Asplund et al. 2009).

\section{RESULTS}

\subsection{Tests of Pixel-phase Correlations}

To investigate the effectiveness of the data detrending we measure the correlations of the signals with the pixel-phase position, before and after the corrections. We refer to the Pearson product-moment correlation coefficient (PCC), 

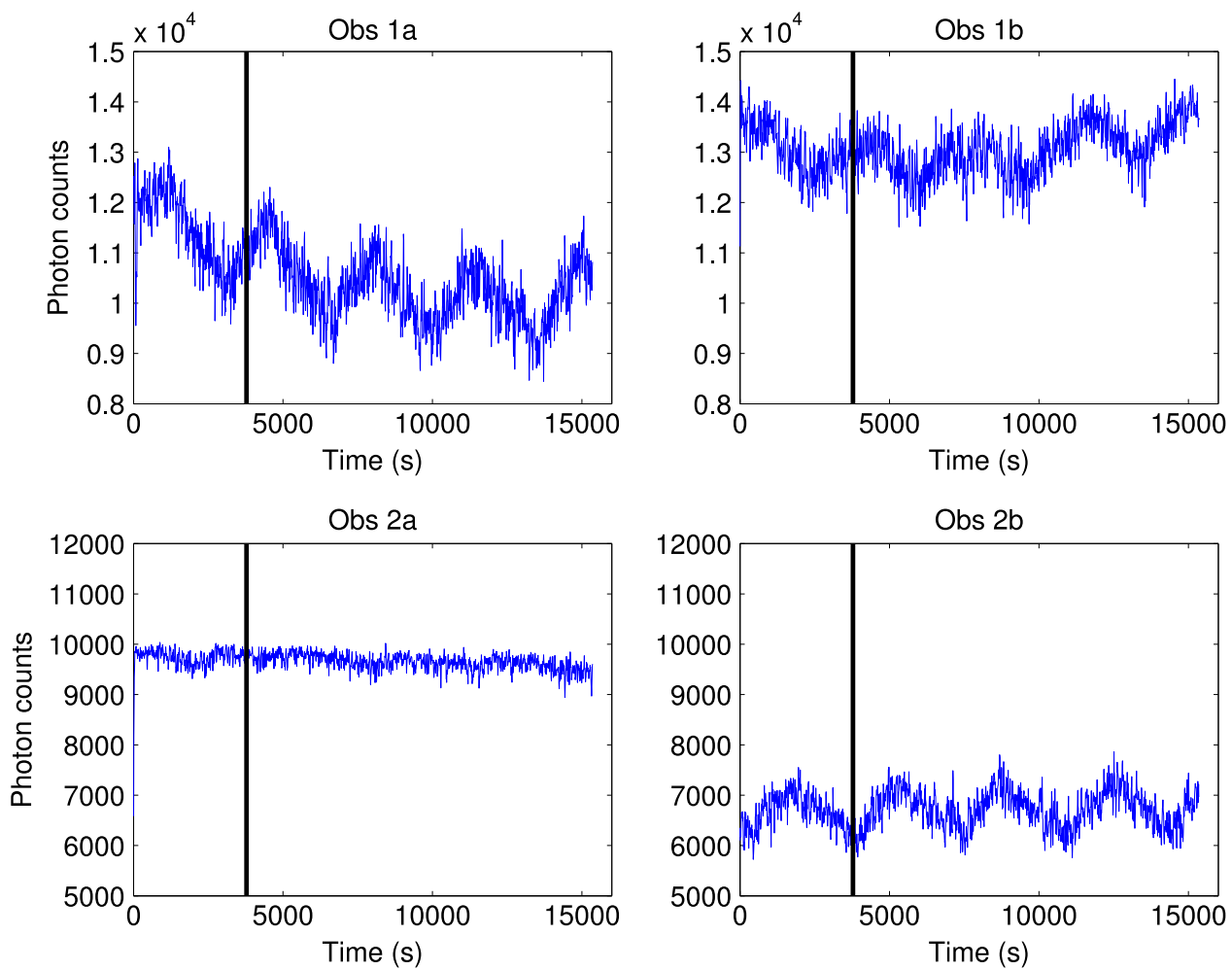

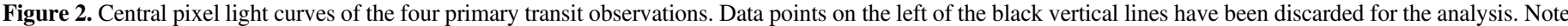
that pixel light curves are dominated by systematics, and the transit signal is not visible by eye (but it is present, as proven by ICA retrieval).
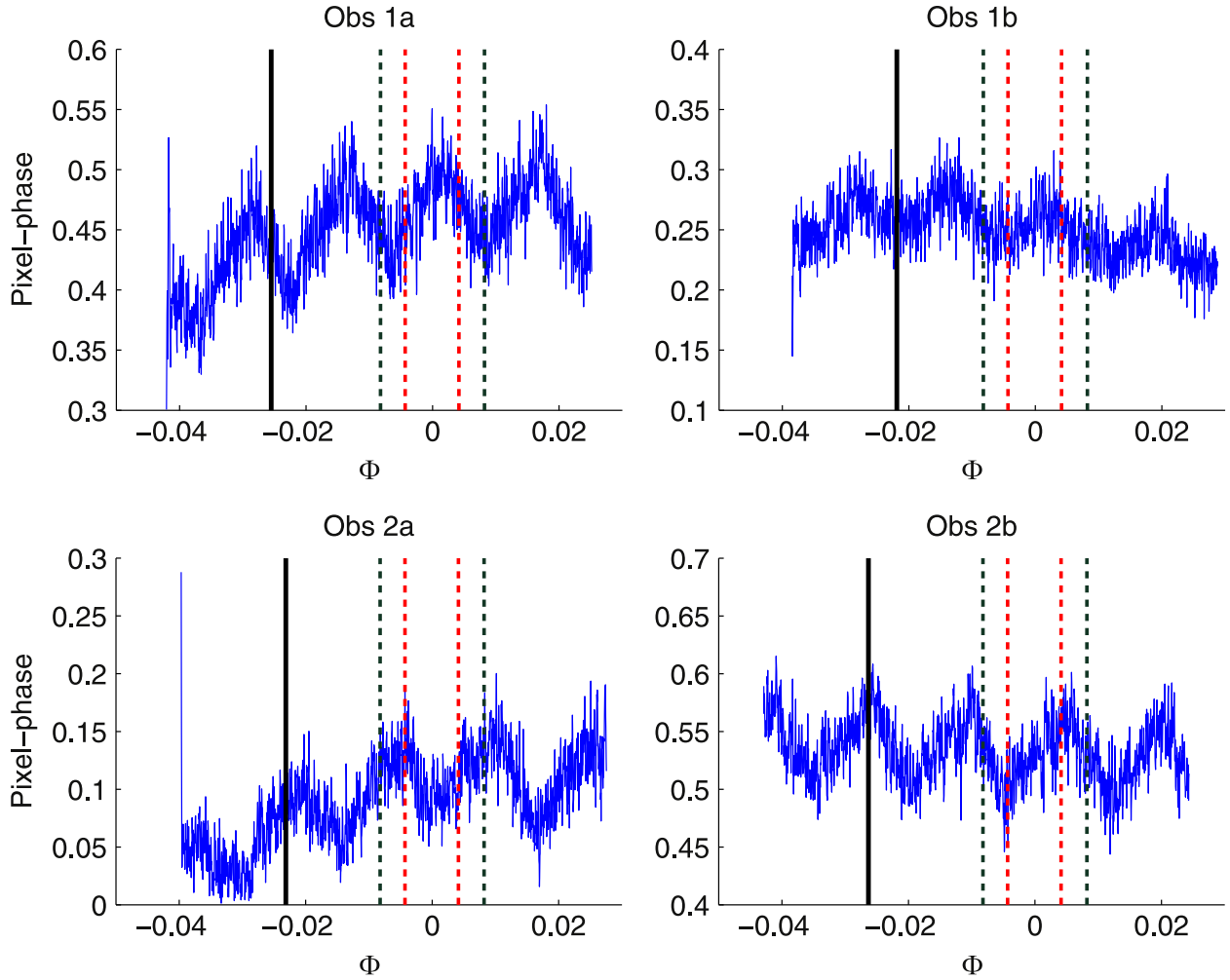

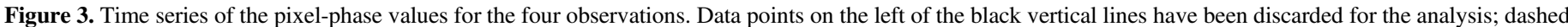
green lines delimit the ends of pre-transits and the beginning of post-transits; dashed red lines delimit the in-transits. 

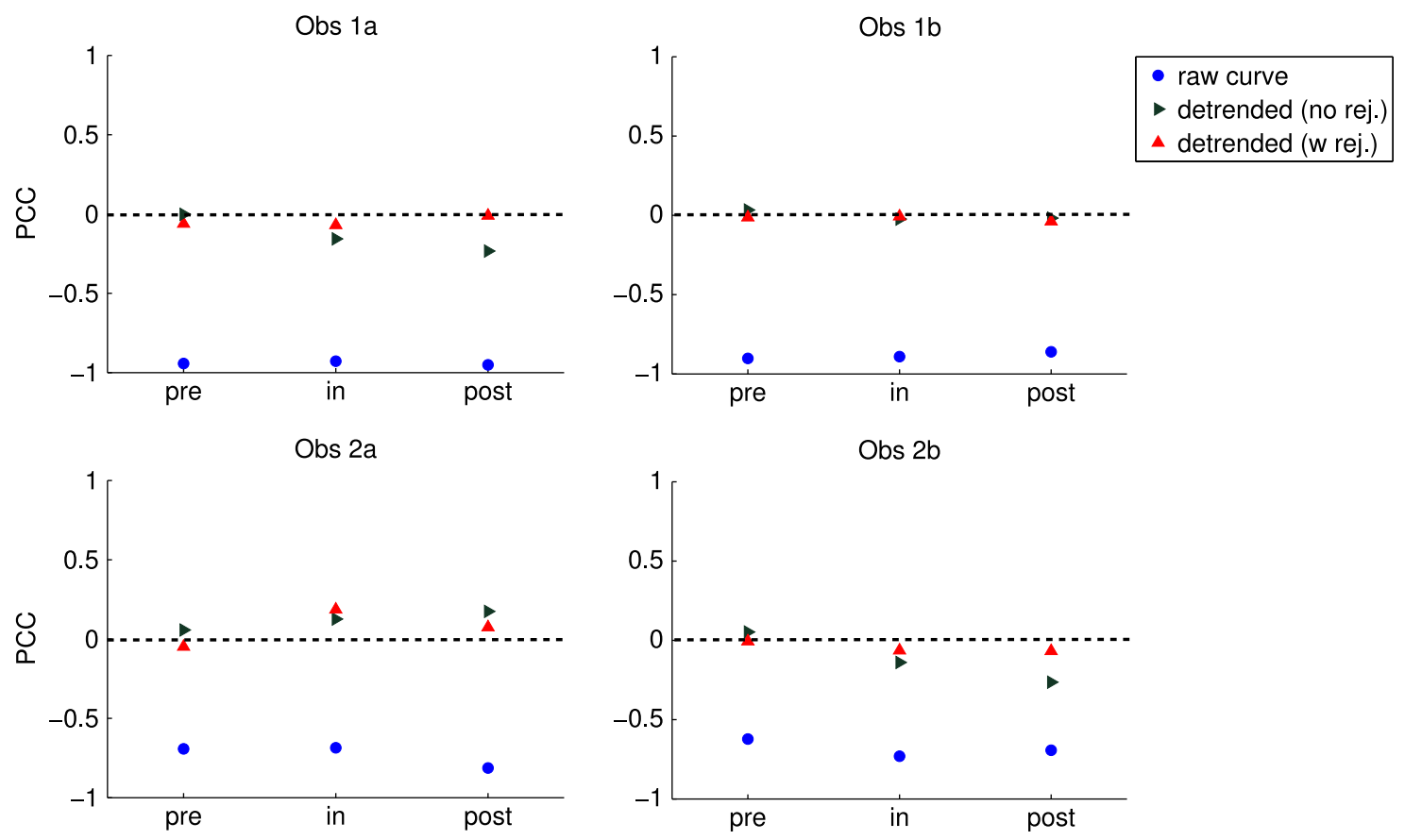

Figure 4. PCCs between fluxes and pixel-phases for pre-, in-, and post-transits of the four light curves. Blue circles indicate raw data, green rightwards triangles indicate ICA detrended data with no rejections, and red upwards triangles indicate ICA detrended data after rejecting the first 450 points.

Obs 1a
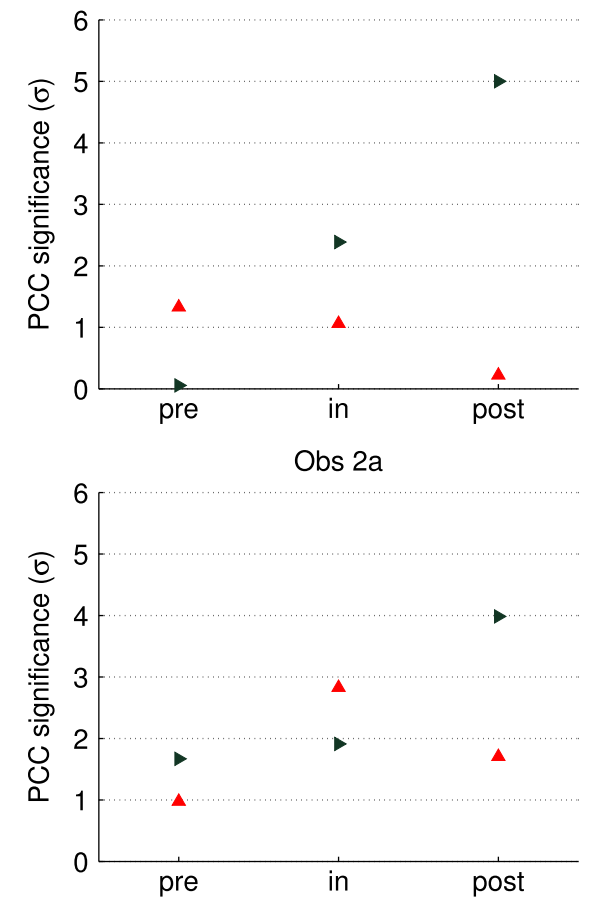

Obs $1 b$
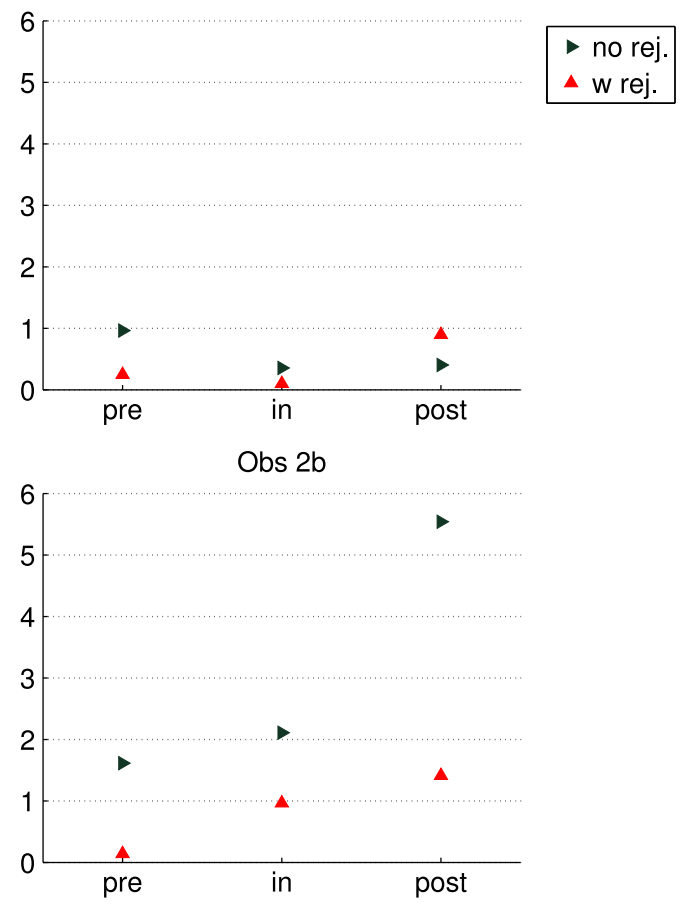

Figure 5. Significance level of correlation between fluxes and pixel-phases for the four observations. Green rightwards triangles indicate ICA detrended data with no rejections and red upwards triangles indicate ICA detrended data after rejecting the first 450 points.

defined as:

$$
\mathrm{PCC}=\frac{\operatorname{cov}(X, Y)}{\sigma_{X} \sigma_{Y}}
$$

where $\operatorname{cov}(X, Y)$ is the covariance of the signals $X$ and $Y$, and $\sigma_{X}$ and $\sigma_{Y}$ are the standard deviations. In this context, $X$ and $Y$ are temporal series of fluxes and pixel-phases. The PCCs are measured over three intervals: pre-, in-, and post-transit, where the astrophysical signals are expected to be almost flat. ${ }^{5}$ In general $-1 \leqslant \mathrm{PCC} \leqslant+1$, where +1 is total positive correlation, -1 is total negative correlation, and 0 is no correlation.

\footnotetext{
5 We used the following definitions: pre-transit $(\phi<-0.0082)$; in-transit $(-0.00433<\phi<0.00416)$; post-transit $(\phi>0.0082)$. These have been decided so that all the transit models obtained during the analysis, modified with no limb darkening, are flat in these three intervals. We checked that other reasonable choices of the limits do not affect this analysis.
} 

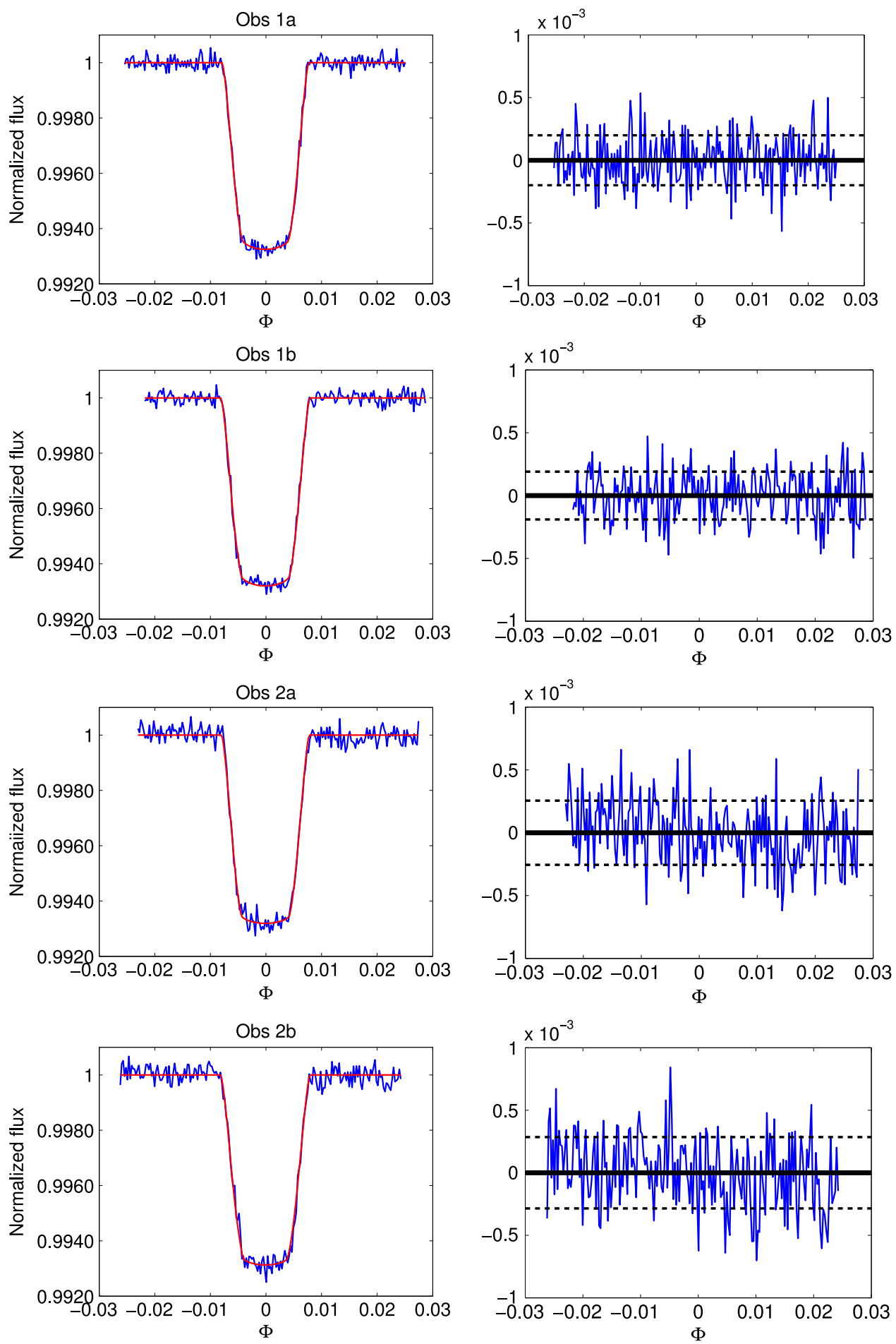

Figure 6. Left panels: (blue) detrended light curves for the four observations with (red) best transit models overplotted, binned over seven points; best transit models are calculated with $p, a_{0}$, and $i$ as free parameters, and Phoenix quadratic limb darkening coefficients (see Section 2.3.1). Right panels: residuals between detrended light curves and best transit models; the black horizontal dashed lines indicate the standard deviations of residuals.

Figure 3 reports the temporal series of pixel-phases. Figure 4 reports the values of the PCCs measured on the pre-, in-, and post-transit for each observation, uncorrected, and corrected without and with pre-transit truncation. The original data are strongly anticorrelated with the pixel-phase, with PCC $\lesssim-0.9$ for channel 1, and PCC $\sim-0.7$ for channel 2. After the ICA detrending including all the data, these correlations are significatively reduced $(|\mathrm{PCC}|<0.3)$. If we remove the first
450 data points, the ICA detrending generally performs significantly better $\left(|\mathrm{PCC}| \sim 10^{-3}-7 \times 10^{-2}\right)$. Figure 5 reports the level of significance of the residual correlations in the detrended data, calculated with a permutation test. When we reject the first 450 data points, the residual correlations in the detrended data are below $1.5 \sigma$, except for Obs. 2a, for which the residual correlation is higher in any case. The residual correlations without the cut of the first 450 data points are 
Table 3

Transit Parameter Estimates for the Four Observations, by Fitting $p, a_{0}$, and $i$ as Free Parameters, with ATLAS Quadratic Limb Darkening Coefficients (Table 2)

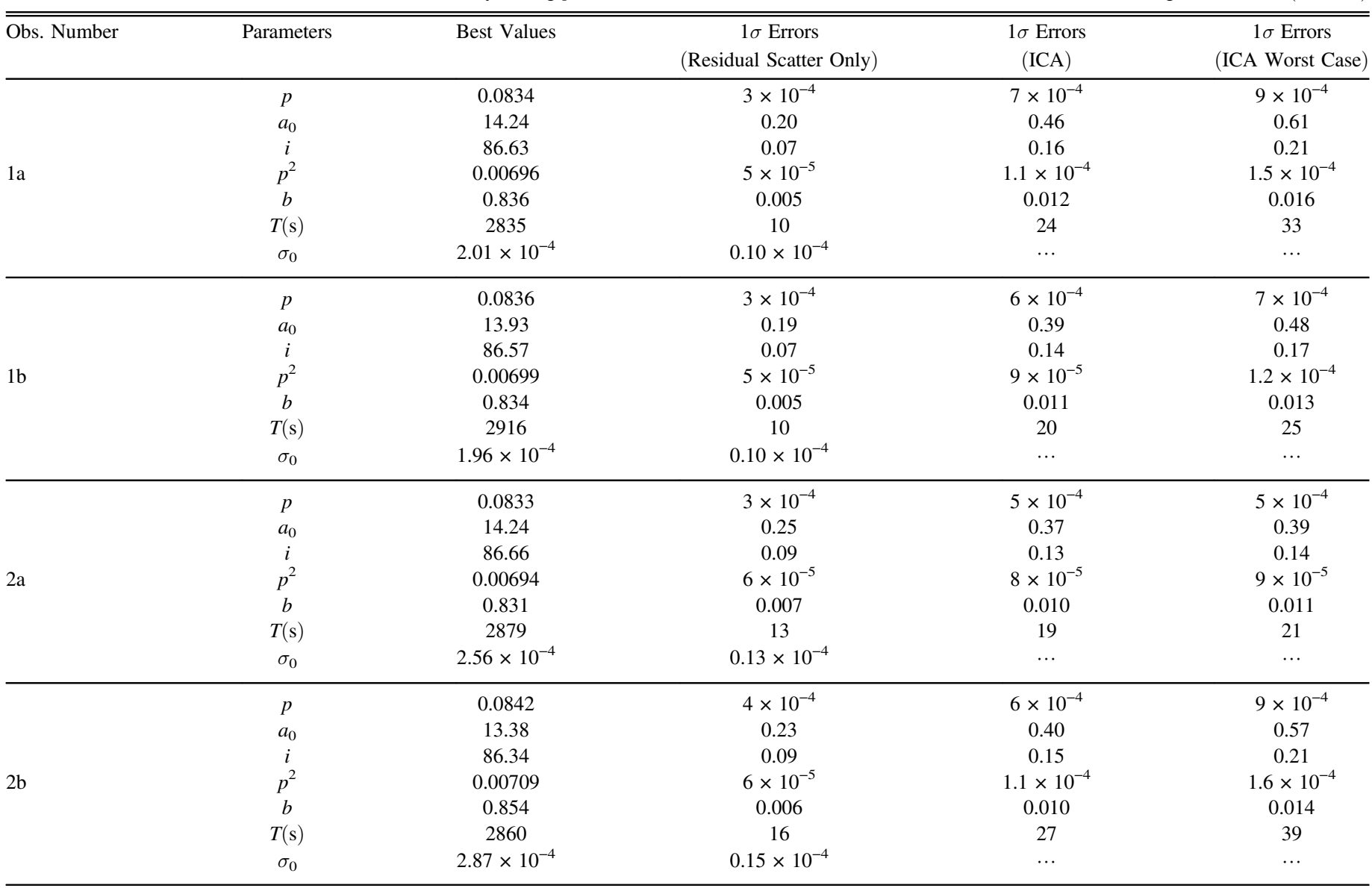

Note. We report the partial error bars obtained by the residuals, the final error bars, and the worst-case error bars (Equation (1), Section 2.2, and Appendix A.3).

larger: $>4 \sigma$ in the post transit, with the exception of Obs. $1 \mathrm{~b}$, for which the residual correlations are below $1 \sigma$ in any case.

\subsection{Fitting $p, a_{0}$, and $i$}

Figure 6 reports the detrended light curves, binned over seven points, with the relative best transit models, and the residuals. The transit models in Figure 6 are computed with $\gamma_{1}$ and $\gamma_{2}$ Phoenix coefficients. Analogous transit models computed with $\gamma_{1}$ and $\gamma_{2}$ Atlas coefficients are very similar, with average standard deviations $\lesssim 1.9 \times 10^{-5}$, and maximum discrepacies $\lesssim 10^{-4}$. Discrepancies between the transit models and the detrended light curves are at the level $\sim 2.0 \times 10^{-4}$ for IRAC channel 1 , and $\sim 2.6-2.9 \times 10^{-4}$ for IRAC channel 2 , therefore it is not possible to distinguish between Atlas and Phoenix models from the data. Best parameter results and error bars are reported in Figure 7, and in Tables 3 and 4. Atlas and Phoenix stellar models lead to two systematically different parameter sets, but both are within the error bars. All the parameters from different observations are comparable within $1 \sigma$, even neglecting the detrending errors $\left(\sigma_{\text {ICA }}\right)$, except the transit durations for Obs $1 \mathrm{a}$ and $1 \mathrm{~b}$. This is discussed in the following sections.

\subsubsection{Combining Observations}

We performed two couples of simultaneos fits, one for the $3.6 \mu \mathrm{m}$ and one for the $4.5 \mu \mathrm{m}$ light curves, with Atlas and
Phoenix limb darkening coefficients, assuming common orbital parameters $\left(a_{0}\right.$ and $\left.i\right)$, and potentially different transit depths $(p)$, in order to cancel the effects of parameter intercorrelations. The assumption that orbital parameters are the same during each observation is very reliable, because they are sparse over a short period of time (less than one month, or nine planetary orbital periods), so that variations due to relativistic effects, external perturbers, or tidal effects would be very small compared to the error bars (Alonso et al. 2008; Jordán \& Bakos 2008; Pál \& Kocsis 2008).

The results of these combined fits are reported in Figures 8 and 9, and in Tables 5 and 6. The $4.5 \mu \mathrm{m}$ transit depths become identical, with an intermediate value between the two determined with separate fits; the $3.6 \mu \mathrm{m}$ transit depths slightly diverges, but their separation is still less than $1 \sigma$. The standard deviations of residuals between the detrended light curves and the transit models increase $\sim 2-3 \times 10^{-6}$ for Obs $2 \mathrm{a}$ and $2 \mathrm{~b}$ (negligible), and $\sim 7-8 \times 10^{-6}$ for Obs $1 \mathrm{a}$ and $1 \mathrm{~b}$ (comparable with the $\sigma_{0}$ uncertainties). The assumption of common orbital parameters for Obs $2 \mathrm{a}$ and $2 \mathrm{~b}$ may be valid, being the consequent transit models as good as the individually fitted ones. With the transit depths also being identical, the two light curves are very well approximated by the same transit model. The original discrepancies between the two sets of transit parameters were enlarged by their intercorrelations. The same assumption for Obs 1a and 1b lead to worse transit models and 
Table 4

Transit Parameter Estimates for the Four Observations, by Fitting $p, a_{0}$, and $i$ as Free Parameters, with PHOENIX Quadratic Limb Darkening Coefficients (Table 2)

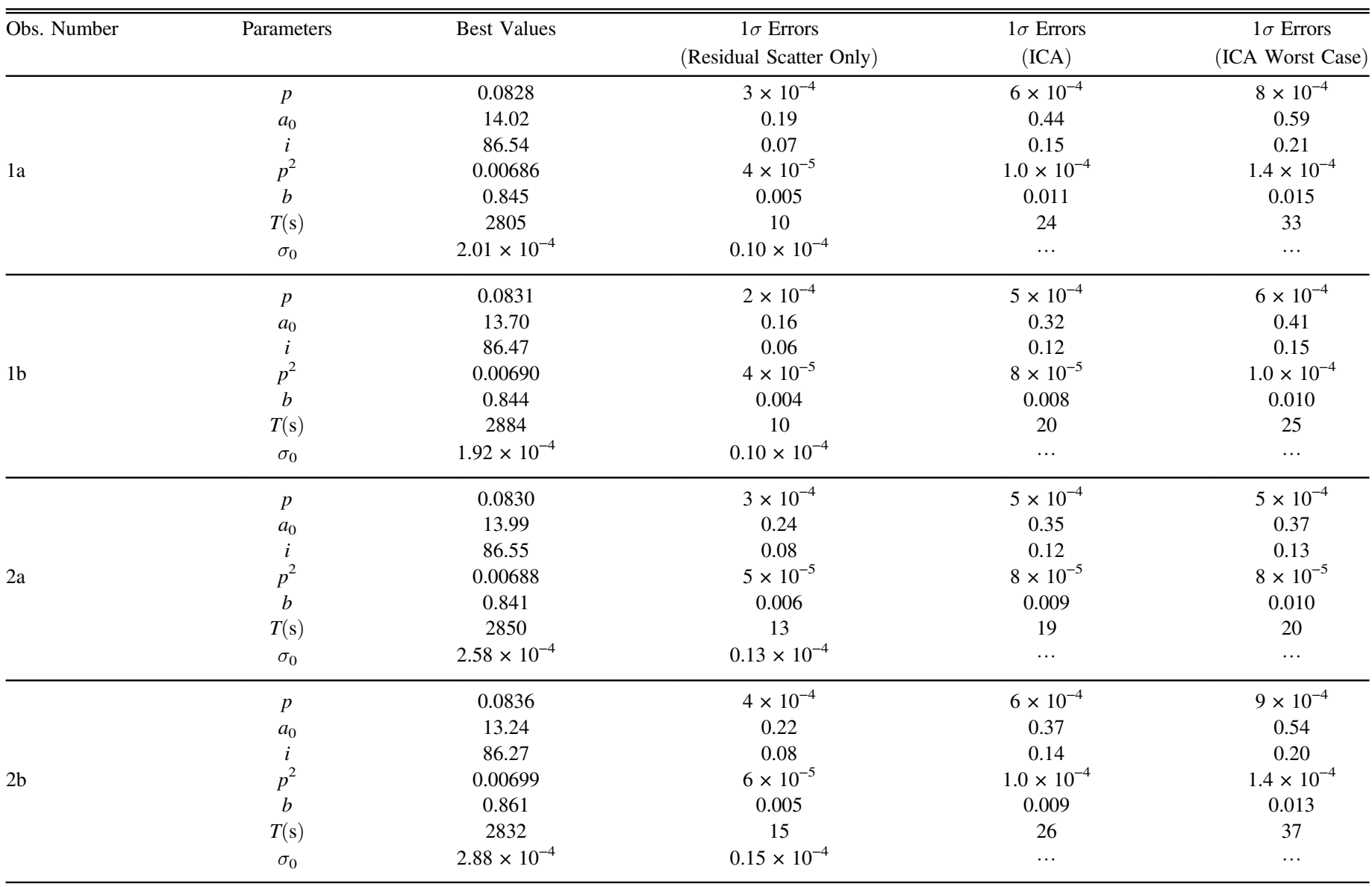

Note. We report the partial error bars obtained by the residuals, the final error bars, and the worst-case error bars (Equation (1), Section 2.2, and Appendix A.3).

more divergent transit depths, but, in both cases, not dramatically.

\subsection{Timing Variations}

We performed transit model-fits with a free phase-shift, in addition to $p, a_{0}$, and $i$, in order to investigate the effect of possible timing variations. Figure 10 reports the time-shifts obtained. No evidence of timing variation has been detected, with upper limits <30 s. Both Atlas and Phoenix stellar models lead to the same shifts. Other parameter estimates are not affected.

\section{DISCUSSION}

\subsection{Comparing Observations}

Figures 11 and 12 report the superpositions of 3.6 and 4.5 $\mu \mathrm{m}$ light curves, respectively, and the residuals. In both cases the mean value of the in-transit residuals is small $\left(\lesssim 5 \times 10^{-5}\right)$, but transit $1 \mathrm{~b}$ is clearly longer than transit $1 \mathrm{a}$, as measured by transit duration $(T)$ parameters. As $T$ is function of the orbital parameters and stellar model, this is the reason why simultaneous fits with common orbital parameters and stellar models do not behave very well. We also note that the ingresses of transits $2 \mathrm{a}$ and $2 \mathrm{~b}$ have different slopes.
The difference between $p^{2}$ values at the two wavelengths is (on average) $\sim 5 \times 10^{-5}$, and there is no evidence of differences in planetary atmosphere's absorption at the two wavelengths.

The orbital parameters at the two wavelengths are also comparable, as detailed in Sections 3.2 and 3.2.1. Simultaneous fits over the four observations with common orbital parameters do not add any information.

\subsection{Comparison with Previous Analyses of the Same Observations}

Figure 7 reports the parameter values obtained in this paper for the individual observations with the analogs reported by Beaulieu et al. (2011) and Knutson et al. (2011). Our results suggest a constant value of the transit depth (largely within $1 \sigma$ ) both between the 3.6 and $4.5 \mu \mathrm{m}$ observations, and for the two wavelengths. Knutson et al. (2011) report variations of the transit depth with a $3.4 \sigma$ significance between the two epochs at $3.6 \mu \mathrm{m}$, and $2.1 \sigma$ at $4.5 \mu \mathrm{m}$, which they attributed to stellar activity. Beaulieu et al. (2011) also obtained significant differences between different epochs at the same wavelength, but they attributed such discrepancies to an unfavorable transit-systematic phasing, then they discarded those epochs from the analysis. Our error bars are generally comparable to the ones reported in both previous papers, but in some cases they are larger by up to a factor $\sim 2$. This is not surprising, because we are not making any prior assumptions 

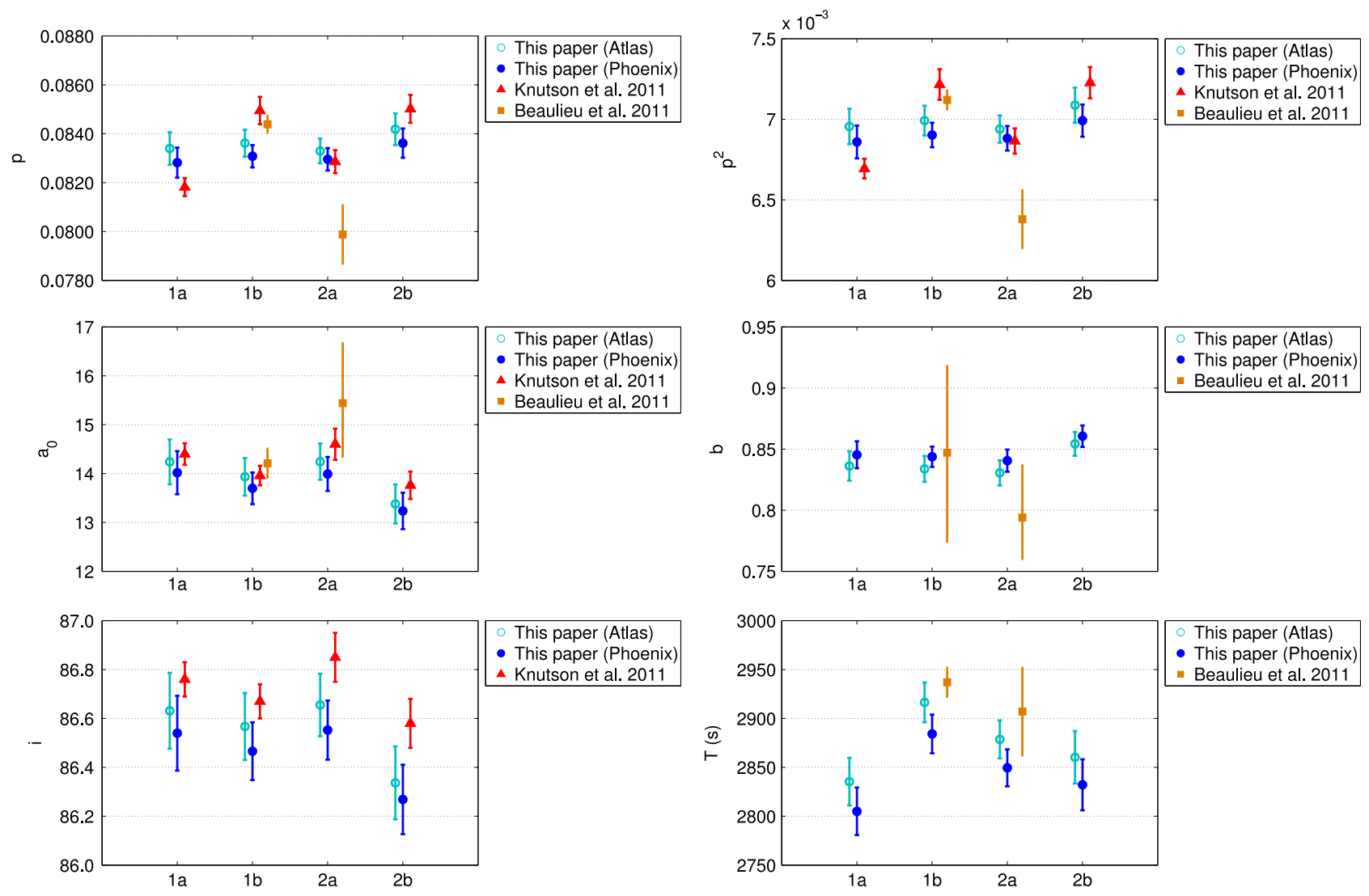

Figure 7. From top to bottom: comparisons of the parameters $p, a_{0}$, and $i$ (left side), and $p^{2}, b$, and $T$ (right side), obtained in this paper with Atlas stellar model (cyan, empty circles), Phoenix stellar model (blue, full circles), in Knutson et al. (2011; red triangles), and in Beaulieu et al. (2011; yellow squares).
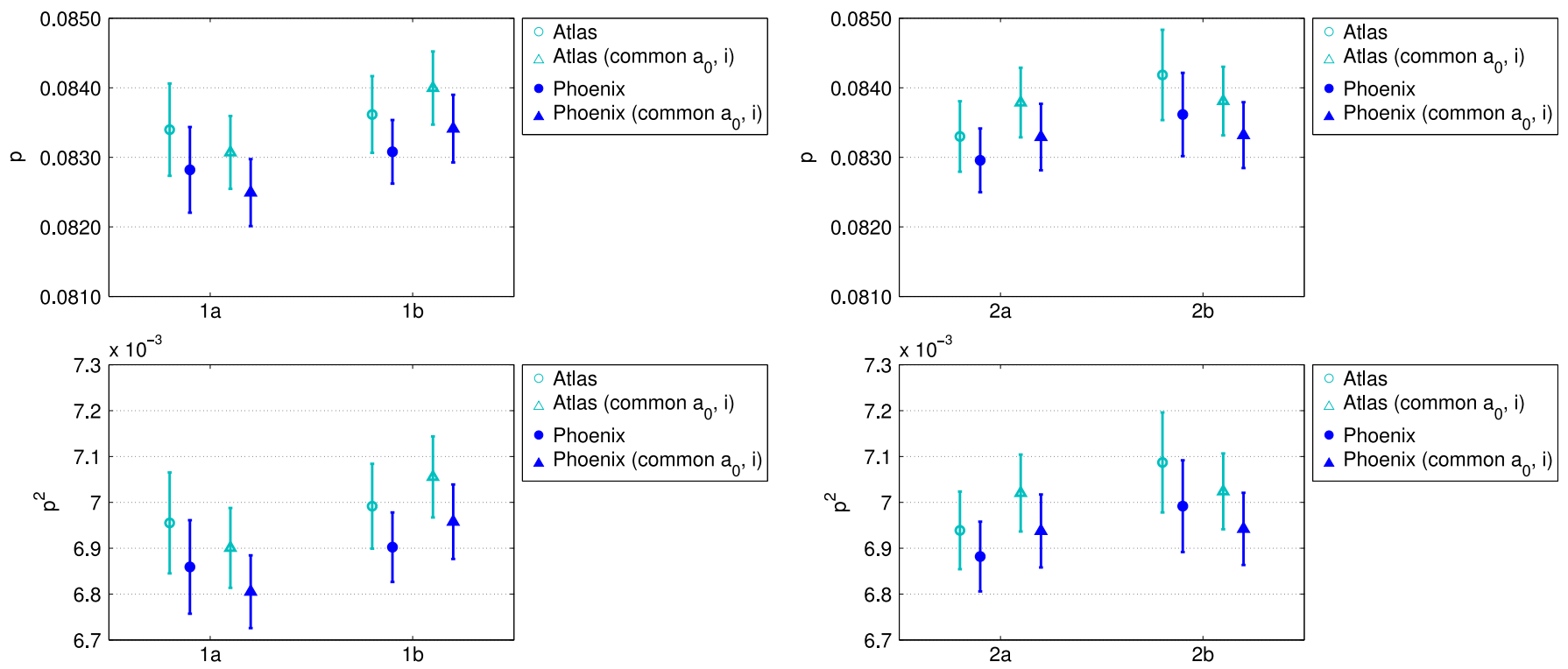

Figure 8. Comparisons of the parameters $p$ and $p^{2}$, obtained in this paper, with common orbital parameters for observations at the same wavelength, and Atlas or Phoenix stellar models.

about the signals to guarantee a high degree of objectivity (Waldmann 2012; Waldmann et al. 2013; Morello et al. 2014). We conclude that our detrending method leads to more robust results than the previous ones in the literature, and they show no evidence of stellar activity variations at $\sim 10^{-4}$ photometric level. Recent results from Hubble/WFC3 observations at 1.2-1.6 $\mu \mathrm{m}$ (Knutson et al. 2014) also show no significant transit depth variations over four observations in about two months.

\subsection{Comparison with Other Observations}

Figure 13 compares our estimated transit depth values at 3.6 and $4.5 \mu \mathrm{m}$ (averaged over the observations at the same 

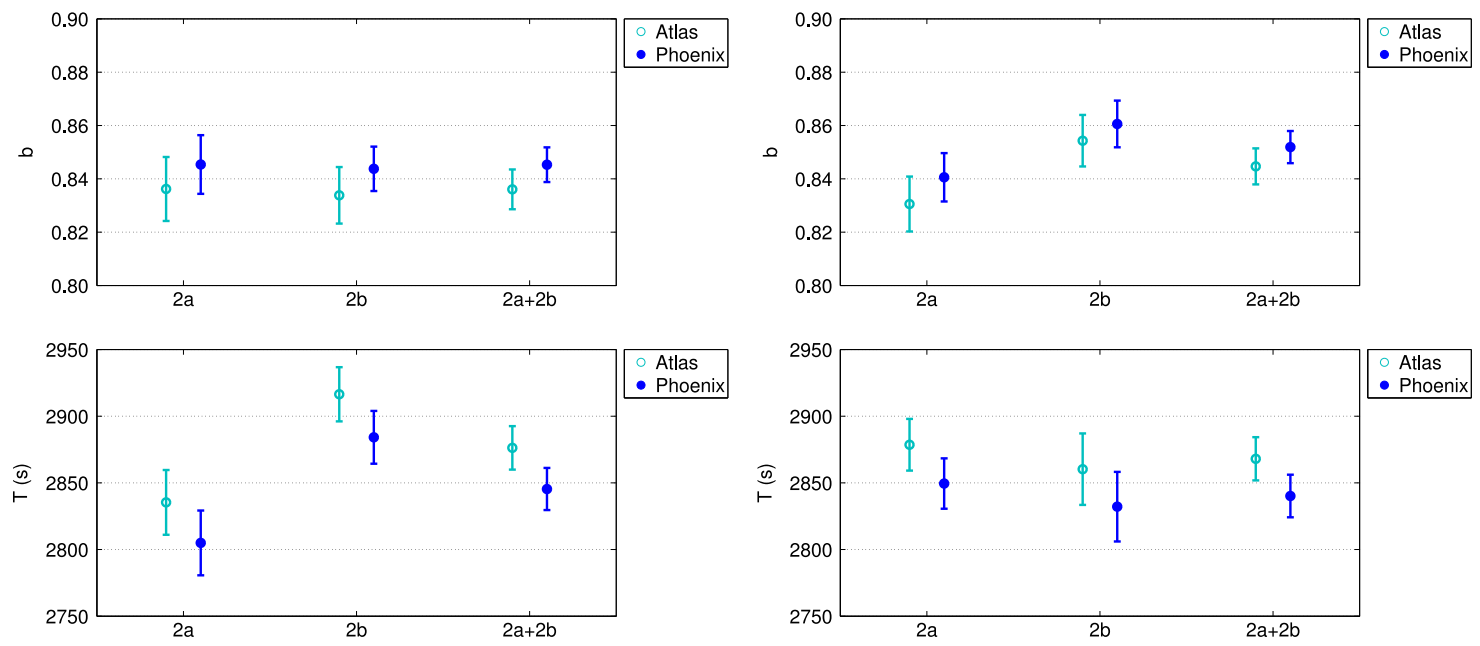

Figure 9. Comparisons of the parameters $b$ and $T$, obtained in this paper, with common orbital parameters for observations at the same wavelength, and Atlas or Phoenix stellar models.

Table 5

Transit Parameter Estimates for the Four Observations, by Fitting $p$ as a Free Parameter, $a_{0}$ and $i$ Identical for the Observations at the Same Wavelength, with ATLAS Quadratic Limb Darkening Coefficients (Table 2)

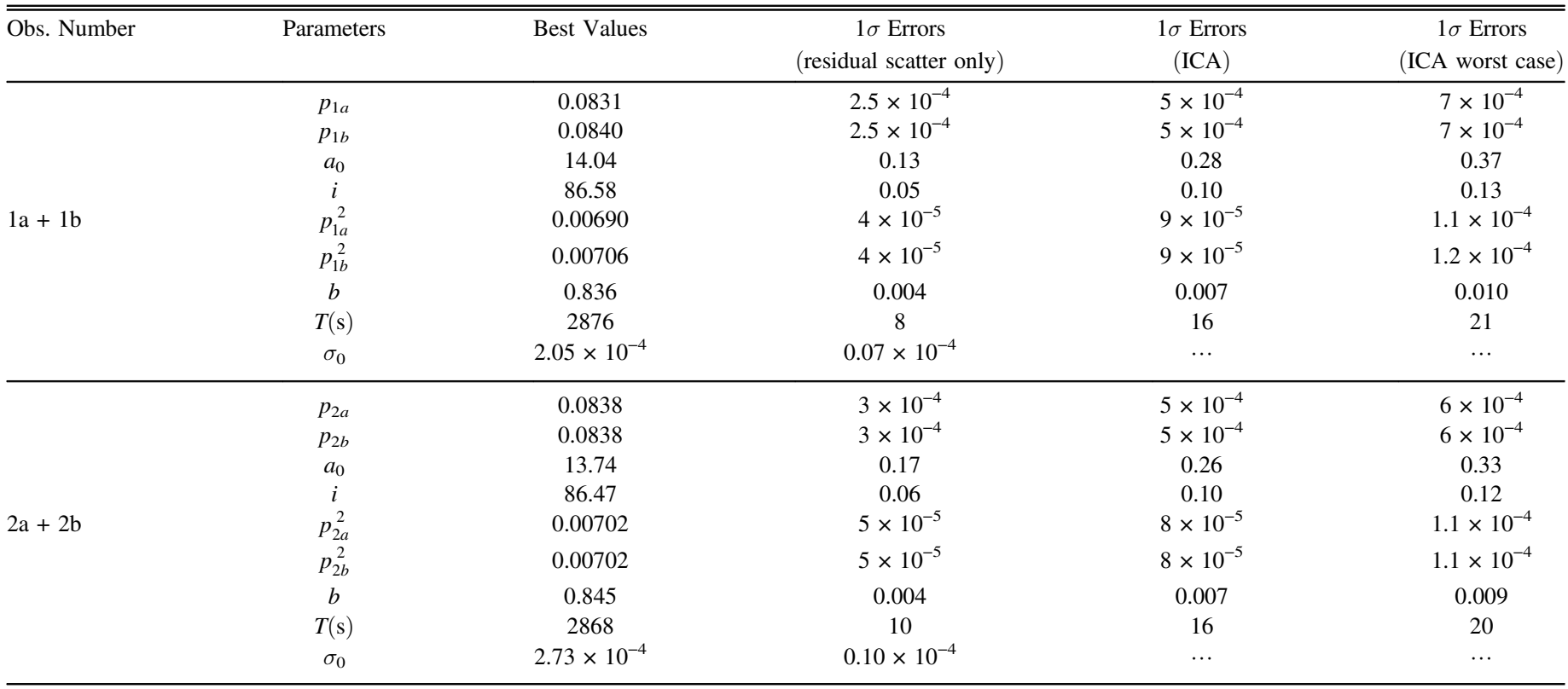

Note. We report the partial error bars obtained by the residuals, the final error bars, and the worst-case error bars (Equation (1), Section 2.2, and Appendix A.3).

wavelength) with the most recent results at $1.2-1.6$ $\mu \mathrm{m}$ (Knutson et al. 2014). The resulting spectrum is featureless within the error bars. However, when comparing transit depth measurements at different wavelengths, we should ensure that the GJ 436 system is uniformly modeled, i.e., the same stellar model and orbital parameters. A uniform multiwavelength reanalysis is required to confirm this result, and investigate potential small features.

Our non-detection of TTVs higher than $\sim 30 \mathrm{~s}$ (see Section 3.3) is consistent with previous analyses in the infrared (Alonso et al. 2008; Cáceres et al. 2009; Pont et al. 2009; Ballard et al. 2010; Knutson et al. 2011, 2014). We measured a significant transit duration variation (TDV; $\sim 80 \mathrm{~s}$ ) between Obs $1 \mathrm{a}$ and $1 \mathrm{~b}$. We did not find any study of TDVs for GJ 436b in the literature, but injecting parameters from Knutson et al. (2011) into our Equation (3) we obtain a similar trend for the same observations. More observations are required to investigate the cause of the apparent TDV between Obs 1a and 1b, whether it is due to a perturber (as currently required to explain the high orbital eccentricity), a stellar phenomenon, or something else.

\section{CONCLUSIONS}

We have applied a blind signal-source separation method, first proposed by Morello et al. (2014), to analyze other photometric data of primary transits of an exoplanet, and extending its validity to the Spitzer/IRAC $4.5 \mu \mathrm{m}$ band. These data sets were more challenging to analyze because of the lower transit depth, comparable with the amplitude of the instrumental pixel-phase signal; the transit duration, very similar to the period of said signal; and possible stellar variability. 
Table 6

Transit Parameter Estimates for the Four Observations, by Fitting $p$ as a Free Parameter, $a_{0}$ and $i$ Identical for the Observations at the Same Wavelength, with PHOENIX Quadratic Limb Darkening Coefficients (Table 2)

\begin{tabular}{|c|c|c|c|c|c|}
\hline Obs. number & Parameters & Best Values & $\begin{array}{c}1 \sigma \text { Errors } \\
\text { (Residual Scatter Only) }\end{array}$ & $\begin{array}{c}1 \sigma \text { Errors } \\
(\text { ICA })\end{array}$ & $\begin{array}{c}1 \sigma \text { Errors } \\
\text { (ICA Worst Case) }\end{array}$ \\
\hline \multirow{7}{*}{$1 a+1 b$} & $p_{1 a}$ & 0.0825 & $2 \times 10^{-4}$ & $5 \times 10^{-4}$ & $6 \times 10^{-4}$ \\
\hline & $p_{1 b}$ & 0.0834 & $2 \times 10^{-4}$ & $5 \times 10^{-4}$ & $6 \times 10^{-4}$ \\
\hline & $i$ & 86.49 & 0.04 & 0.09 & 0.12 \\
\hline & $p_{1 a}^{2}$ & 0.00681 & $4 \times 10^{-5}$ & $8 \times 10^{-5}$ & $1.0 \times 10^{-4}$ \\
\hline & $p_{1 b}^{2}$ & 0.00696 & $4 \times 10^{-5}$ & $8 \times 10^{-5}$ & $1.0 \times 10^{-4}$ \\
\hline & $T(\mathrm{~s})$ & 2845 & 7 & 16 & 21 \\
\hline & $\sigma_{0}$ & $2.04 \times 10^{-4}$ & $0.07 \times 10^{-4}$ & $\cdots$ & $\cdots$ \\
\hline \multirow{6}{*}{$2 a+2 b$} & $p_{2 a}$ & 0.0833 & $3 \times 10^{-4}$ & $5 \times 10^{-4}$ & $6 \times 10^{-4}$ \\
\hline & $p_{2 b}$ & 0.0833 & $3 \times 10^{-4}$ & $5 \times 10^{-4}$ & $6 \times 10^{-4}$ \\
\hline & $a_{0}$ & 13.57 & 0.16 & 0.25 & 0.31 \\
\hline & $i$ & 86.40 & 0.06 & 0.09 & 0.11 \\
\hline & $T(\mathrm{~s})$ & 2840 & 10 & 16 & 20 \\
\hline & $\sigma_{0}$ & $2.74 \times 10^{-4}$ & $0.10 \times 10^{-4}$ & $\cdots$ & $\cdots$ \\
\hline
\end{tabular}

Note. We report the partial error bars obtained by the residuals, the final error bars, and the worst-case error bars (Equation (1), Section 2.2, and Appendix A.3).

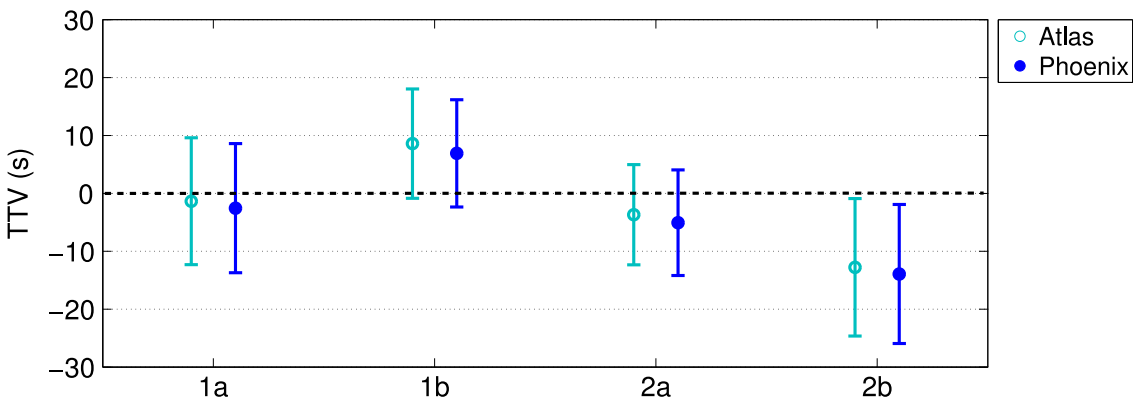

Figure 10. Best fitted time-shifts of the mid-transit with respect to the periodically predicted mid-transit times, assuming Atlas or Phoenix stellar models. Note that results are model-independent, because mid-transit time will not correlate with limb darkening parameters, or any other physical parameters.

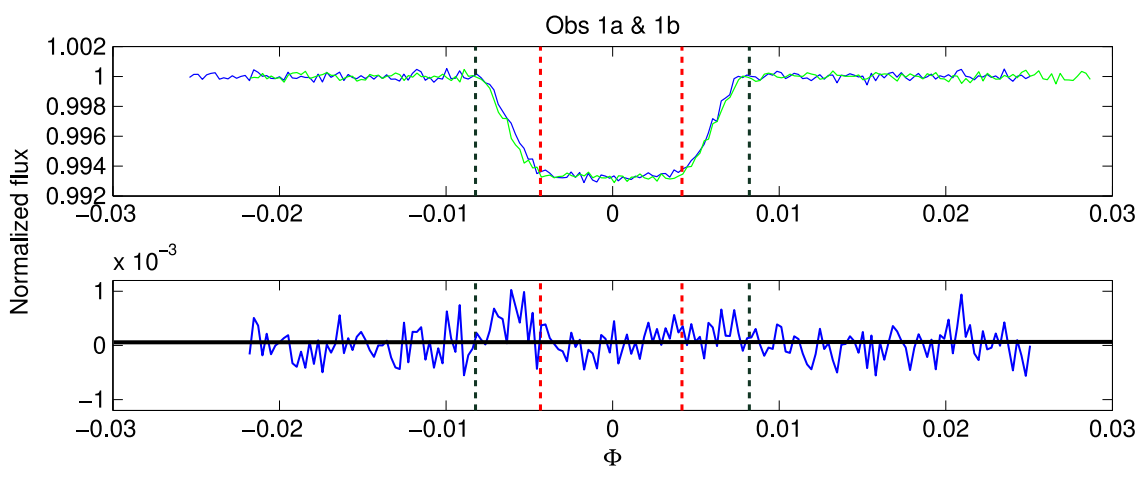

Figure 11. Top panel: detrended light curves for Obs 1a (blue) and Obs $1 \mathrm{~b}$ (green). Bottom panel: residuals between the two observations. Black dotted lines delimit the out-of-transit and red dotted lines delimit the in-transit (as defined in Section 3.1).

We obtain consistent results between transits at different epochs, ruling out stellar activity variations within $\sim 10^{-4}$ photometric level. We do not detect any significant difference for the transit depth at 3.6 and $4.5 \mu \mathrm{m}$, or with the recent measurements at 1.2-1.6 $\mu \mathrm{m}$ (Knutson et al. 2014), supporting the hypothesis of a flat transmission spectrum. We measure a
TDV of $80 \mathrm{~s}$ between transits separated by seven orbits $(2 \sigma$ significance level), but no significant TTVs. More measurements are required to investigate the possible presence of a perturber, and its nature. Also, more uniform analyses at other wavelengths are required to get a more reliable transmission spectrum. 


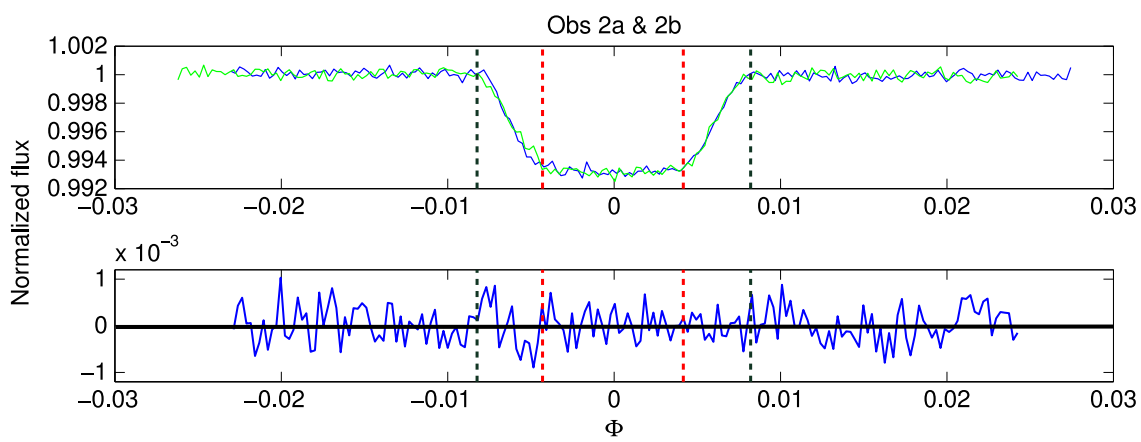

Figure 12. Top panel: detrended light curves for Obs $2 \mathrm{a}$ (blue) and Obs $2 \mathrm{~b}$ (green). Bottom panel: Residuals between the two observations. Black dotted lines delimit the out-of-transit and red dotted lines delimit the in-transit (as defined in Section 3.1).

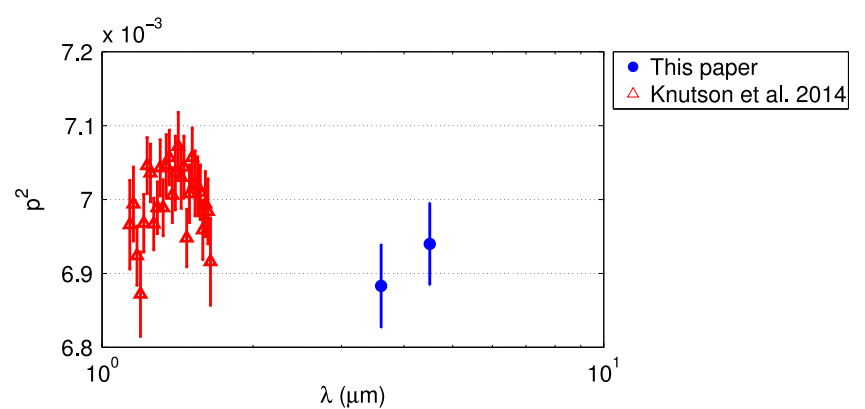

Figure 13. Transit depth values obtained in this paper at 3.6 and $4.5 \mu \mathrm{m}$ (blue circles), and values at $1.2-1.6 \mu \mathrm{m}$ reported by Knutson et al. (2014; red triangles).

G.M. is funded by UCL Perren/Impact scholarship (CJ4M/ CJ0T). I.P.W. is funded by the European Research Council Grant "Exolights." G.T. is funded by the Royal Society. G.M. is supported by "Progetto Premiale-A Way to Other Worlds", funded by the Italian Minister for University and Scientific Research.

\section{APPENDIX A ICA}

\section{A.1 Rationale}

ICA is a special case of "blind source separation" technique: it aims to separate original source signals from observations with minimal assumptions. The assumptions for standard $\mathrm{ICA}^{6}$ are:

1. the source signals are statistically independent;

2. observations are linear mixtures of the source signals;

3. the number of distinguishable observations is not smaller than the number of sources.

The first condition is easily verified if the signals have different origins, i.e., the astronomical target, other background objects, and the instruments. Additionally, some studies found that ICA algorithms can also separate signals that are not exactly independent (Hyvärinen \& Oja 2000; Hyvärinen et al. 2001).

The second condition is more questionable, given that some instrumental systematics might be multiplicative rather than additive. Alternative ICA algorithms consider nonlinear mixing of the source signals, but some additional information is

\footnotetext{
6 There are some variants of ICA that do include prior information, e.g., Barriga et al. (2011), Igual et al. (2002), Stone et al. (2002).
}

required to perform the separation, and, in general, there is not a unique solution (Hyvärinen et al. 2001). Based on the following evidence, we found that, for Spitzer/IRAC light curves, the classic assumption of linear mixing leads to reliable and robust results:

1. detrended light curves present a low level of residual scatter, compared to the literature (Beaulieu et al. 2011; Knutson et al. 2011);

2. planetary and stellar parameters measured at different epochs are consistent (this is not a necessary condition);

3. non-transit components have the same characteristics, e.g., periodicity and amplitude, of known instrumental systematics.

This seems to be in contrast with the standard (empirical) pixel-phase effect method used to detrend Spitzer data (Fazio et al. 2004): flux measurements are correlated with the position of the centroid on a pixel, the cause of this is assumed to be an intra-pixel sensitivity variation, hence the systematics model is multiplied by the astrophysical signal. We are now investigating this question through simulated observations (Morello 2015); we report here our preliminary results:

1. either inter- and intra-pixel effects (or both) can originate systematics similar to the ones observed in Spitzer;

2. inter-pixel effects are additive, as in our ICA model;

3. intra-pixel effects are not additive, but the ICA algorithm is still able to significantly reduce their presence in the light curves (our simulations currently indicate a reduction by a factor of seven for the amplitudes of systematic components from an original $3.5 \times 10^{-3}$ photometric level, outperforming the pixel-phase method by a factor of 2.3-3.3).

The third condition is case dependent, since the number of components is not known a priori, and the number of pixels is limited by the width of the point-spread function. Also, if all the pixels contain the same systematic signals with the same weights relative to the astrophysical signal, the pixel light curves would not be distinguishable, and separation would be impossibile. Given the results we obtained, we infer that we have a sufficient number of distinguishable pixel light curves to detrend our signals up to a $2 \times 10^{-4}$ photometric precision.

\section{A.2 Performances of MULTICOMBI Algorithm}

In this section, we discuss the ability of ICA to separate different kinds of signals. It depends on the particular algorithm used, in our case MULTICOMBI (Tichavský et al. 2008). 

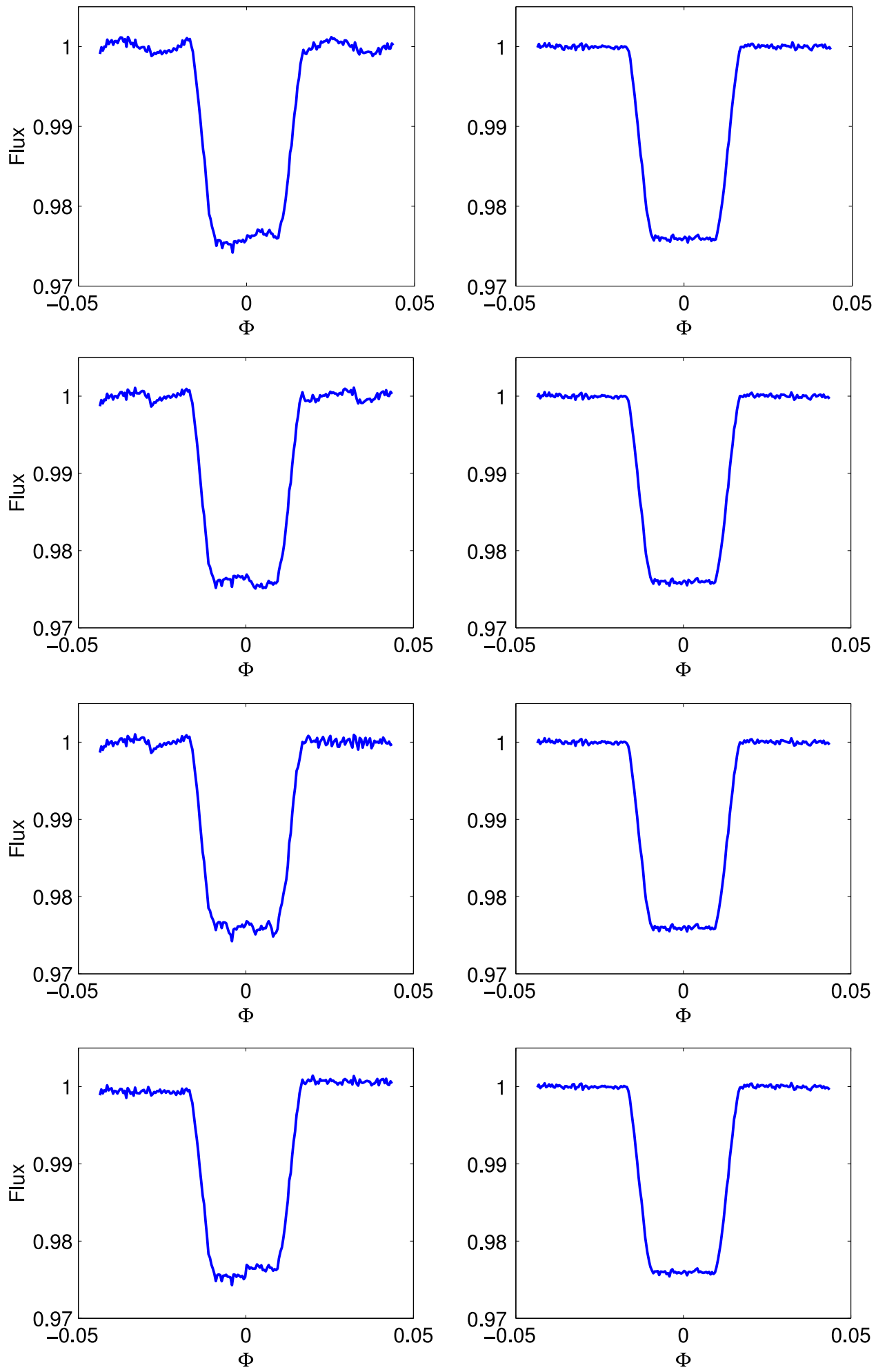

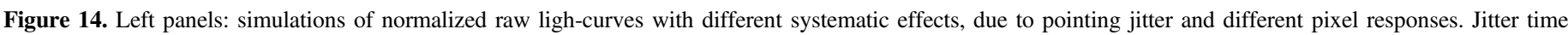

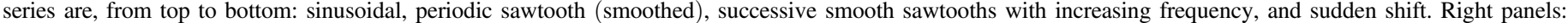
correspondent detrended light curves with pixel-ICA method. Retrieved parameters are consistent with the values adopted to generate the raw light curves.

MULTICOMBI is a powerful tool that optimally mixes two complementary algorithms: EFICA (Koldovský et al. 2006), designed to separate non-Gaussian signals, and WASOBI (Yeredor 2000), specialized to separate Gaussian autoregressive and time-correlated components.
We tested MULTICOMBI performance with simulated observations of planetary transits affected by a large variety of systematic signals, including non-stationary signals with changing frequencies and amplitudes, sudden change points, transient behaviors, and long-term monotonic drifts. In all cases, the algorithm successfully dentrended the systematic 

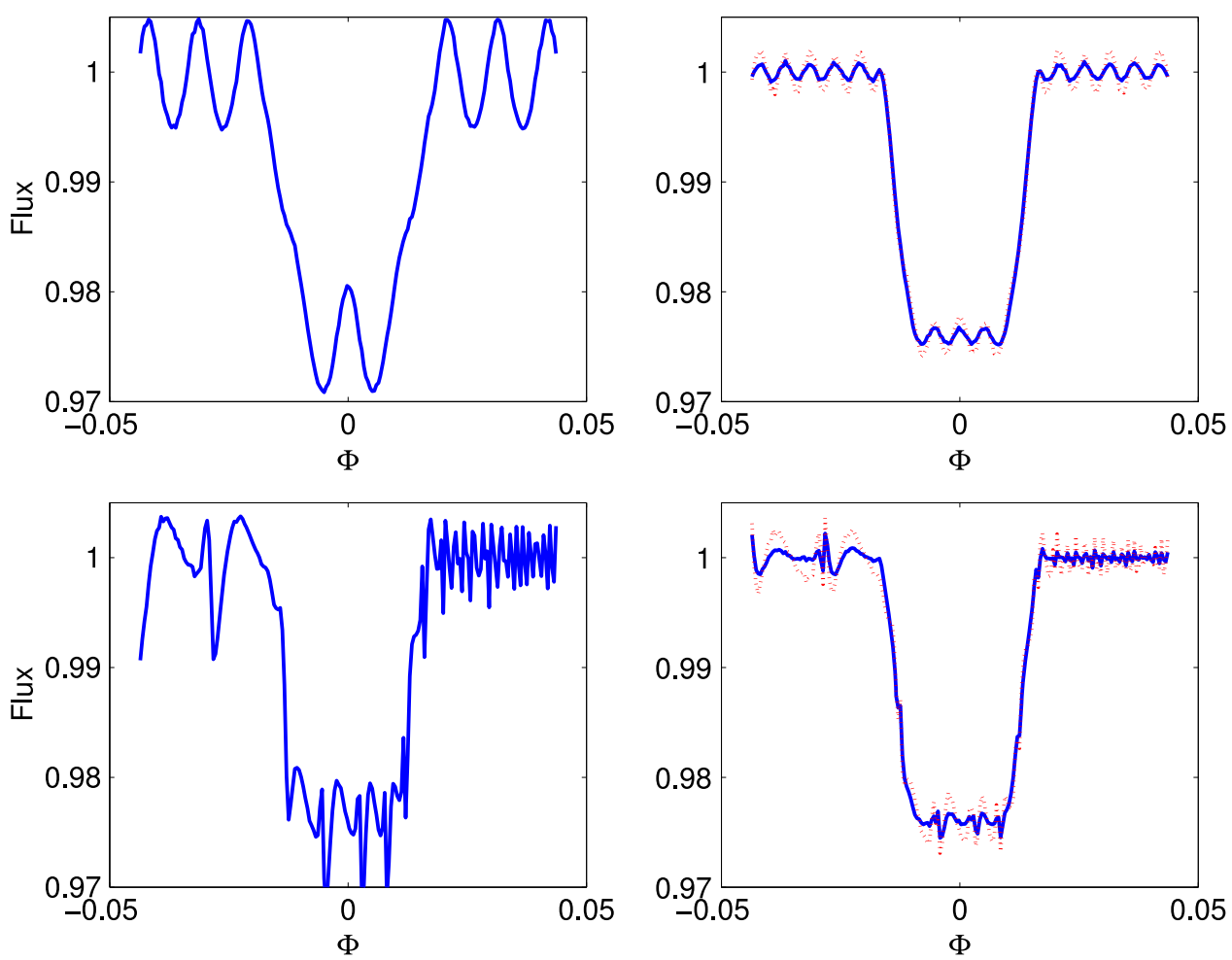

Figure 15. Left panels: simulations of normalized raw ligh-curves with different systematic effects, due to pointing jitter and strong intra-pixel variations. Jitter time series are: (top) sinusoidal and (bottom) successive smooth sawtooths with increasing frequency. Right panels: Correspondent detrended light curves with (blue) pixel-ICA method and (red, dashed) traditional pixel-phase method. Note that ICA method outperforms the pixel-phase method, with lower residual systematics and retrieved parameters closer to the true values.

Table A1

Estimated $\sigma_{\text {ICA }}$ Values for the Four Observations (Equations (A1) and (A2)), and Worst-case Values According to a More Conservative Estimate of ISR (Arithmetic mean of $\mathrm{ISR}^{\mathrm{EF}}$ and $\mathrm{ISR}^{\mathrm{WA}}$ )

\begin{tabular}{lcc}
\hline \hline Obs. Number & $\sigma_{\text {ICA }}$ & $\sigma_{\text {ICA }}(\max )$ \\
\hline $1 \mathrm{a}$ & $4.24 \times 10^{-4}$ & $5.97 \times 10^{-4}$ \\
$1 \mathrm{~b}$ & $3.41 \times 10^{-4}$ & $4.52 \times 10^{-4}$ \\
$2 \mathrm{a}$ & $2.79 \times 10^{-4}$ & $3.07 \times 10^{-4}$ \\
$2 \mathrm{~b}$ & $3.97 \times 10^{-4}$ & $6.42 \times 10^{-4}$ \\
\hline
\end{tabular}

components, except monotonic drifts (see Figures 14 and 15 for some examples). Spitzer/IRAC light curves typically start with a drift before stabilization, which would explain the improved performance if rejecting part of the earlier data points.

A more detailed analysis of the performances of the ICA detrending method adopted in this paper for different instrument cases, i.e., inter- or intra-pixel effects, amplitude, frequency, temporal structure, and non-stationarity of the systematics, individual pixel peculiarities, is ongoing (Morello 2015).

The calculation time for a MULTICOMBI session is, in our cases, less than $3 \mathrm{~s}$. We are considering a number of signals $d=25$, and $N \sim 10^{3}$ data points. For non-binned data sets, i.e., $N \sim 10^{5}$ data points, the calculation time varies in the range 45-105 s, then it is, in general, case dependent. We measured the computation time for different values of $d$ and $N$, and found that the algorithm complexity scales as $\mathcal{O}\left(d^{2} N\right)$, as predicted for EFICA in Koldovský et al. (2006).

\section{A.3 ICA Errors}

If ICA was able to separate the original source signals perfectly, the parameter error bars would be fully determined by the residual scatter on the detrended light-curve. In general, we do not expect this to be the case, since any detrending method would introduce some bias in the parameter estimates. We model such unknown bias as an additive uncertainty, $\sigma_{\text {ICA }}$, in the time series, leading to Equation (1). Morello et al. (2014) report the following formula for $\sigma_{\mathrm{ICA}}$ :

$$
\sigma_{\mathrm{ICA}}^{2}=f^{2}\left(\sum_{j} o_{j}^{2} \mathrm{ISR}_{j}+\sigma_{n t c-f i t}^{2}\right)
$$

where ISR is the so-called Interference-to-signal-ratio matrix, $o_{j}$ are the coefficients of the non-transit-components, $m$ is their number, $\sigma_{\text {ntc }}$ - fit is the standard deviation of residuals from the referent raw light-curve, out of the transit, and $f$ is the normalizing factor for the detrended light-curve. The sum on the left takes into account the precision of the components extracted by the algorithm; $\sigma_{\text {ntc }- \text { fit }}$ indicates how well the linear combination of components approximates the out-of-transit. Note that, while the first term increases with the number of components considered (see Section 2.2), the second term decreases. The optimal strategy is to remove all the extracted non-transit components from the raw light-curve, though many results obtained by removing the most significant components (to be determined) are almost identical (see Morello et al. 2014, Section 2.5.2).

MULTICOMBI code produces two Interference-to-SignalRatio matrices, ISR ${ }^{\mathrm{EF}}$, associated with the algorithm EFICA, and ISR $^{\mathrm{WA}}$, associated with the algorithm WASOBI. In Morello et al. (2014) we estimated the global ISR as the 

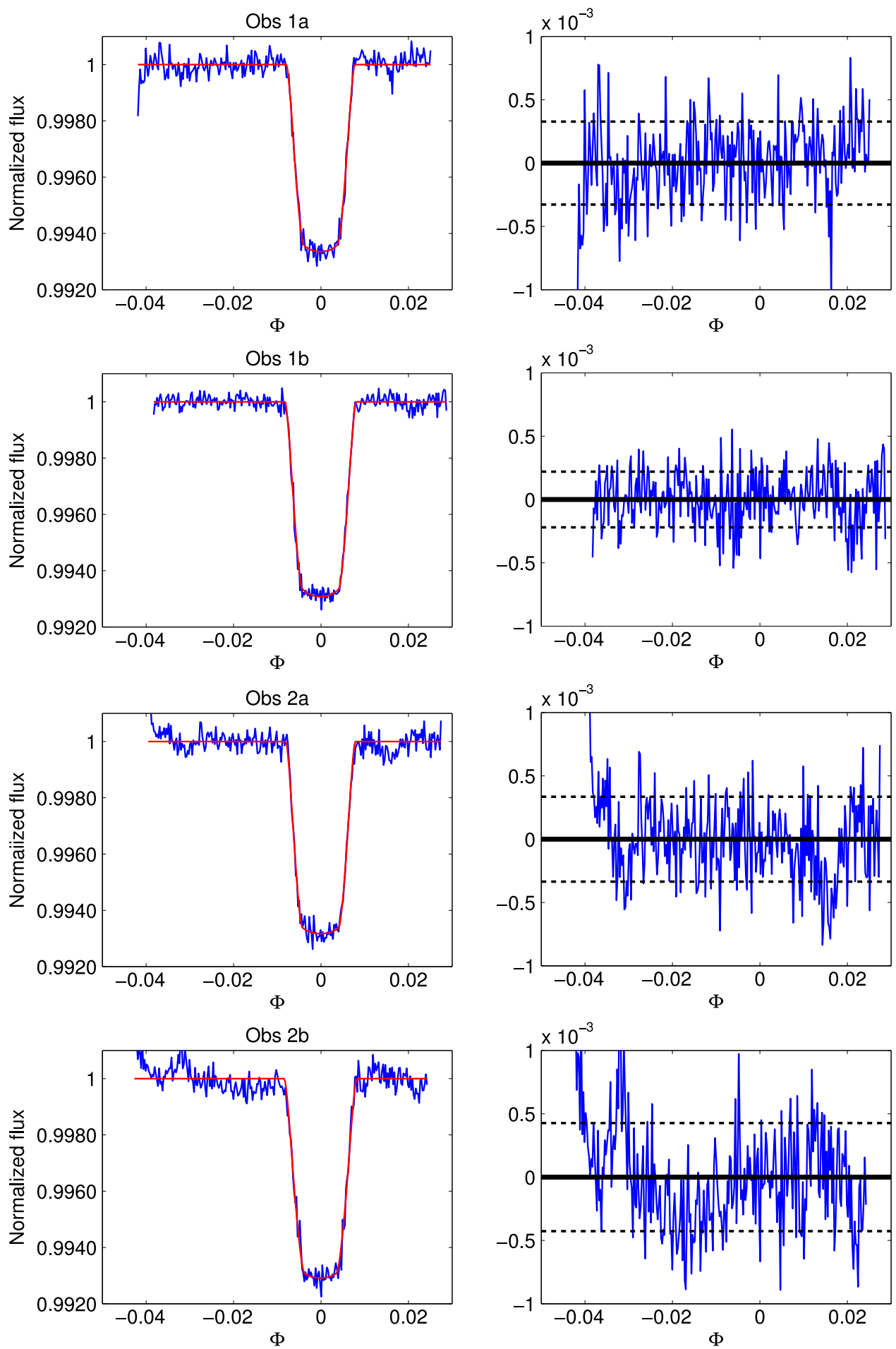

Figure 16. Left panels: (blue) detrended light curves for the four observations without data rejection and (red) best transit models overplotted, binned over seven points. Best transit models are calculated with $p, a_{0}$, and $i$ as free parameters, and Phoenix quadratic limb darkening coefficients (see Section 2.3.1). Right panels: residuals between detrended light curves and best transit models. Black horizontal dashed lines indicate the standard deviations of residuals.

arithmetic mean of ISR ${ }^{\mathrm{EF}}$ and ISR ${ }^{\mathrm{WA}}$. This a very conservative estimate that does not take into account the outperforming separation capabilities of MULTICOMBI compared to EFICA and WASOBI. Here we suggest a more appropriate definition:

$$
\mathrm{ISR}_{i, j}=\min \left(\mathrm{ISR}_{i, j}^{\mathrm{EF}}, \mathrm{ISR}_{i, j}^{\mathrm{WA}}\right) .
$$

In the cases analyzed in Morello et al. (2014), the contributions of the ISR terms were $\sim 10 \%$ of the total error bars, thus adopting the new definition of ISR would not modify the results significantly. Here, we find that the ISR contributions to the error bars are comparable with the other terms, probably because some instrumental systematics and the transit signals have similar timescales and amplitudes, making the separation more uncertain. Table A1 reports the values of $\sigma_{\text {ICA }}$ obtained for each observation, with ISR calculated according to Equation (A2), and according to the arithmetic mean definition. 


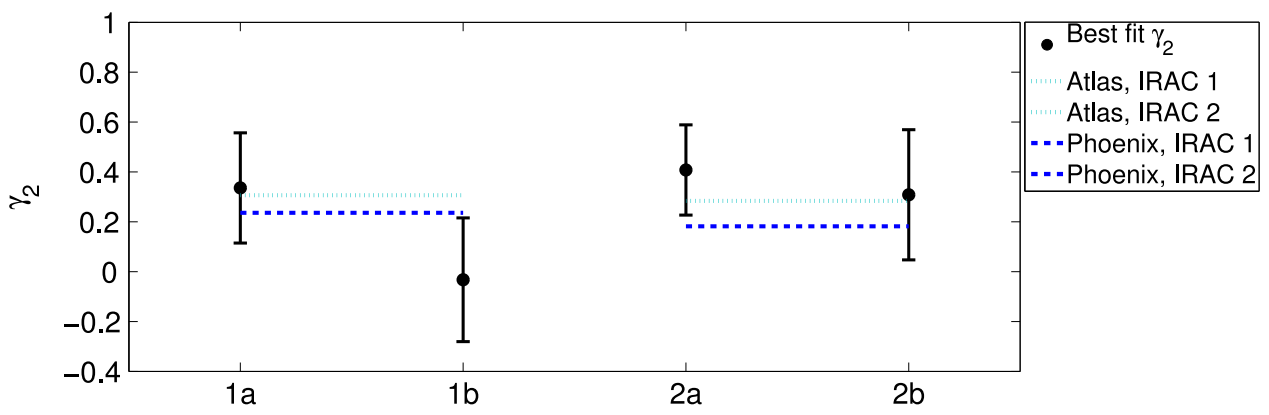

Figure 17. Best fitted values of the quadratic limb darkening coefficient $\left(\gamma_{2}\right)$ for the four observations, assuming $\gamma_{1}=0$.

The error bars obtained in this paper with the definition in Equation (A2) are consistent with results from the same tests of robustness reported in Morello et al. (2014), i.e., different number of components, pixel arrays, and partial time series. For completeness, the tables in Appendix D report the error bars obtained from residual scatter only, including $\sigma_{\text {ICA }}$ with ISR defined as in Equation (A2), and with the previous definition of ISR. The relative difference between error bars obtained with the two definitions of ISR is $0-30 \%$, which, in general, may be important for atmospheric characterization, but does not affect the conclusions obtained in this paper.

\section{APPENDIX B \\ FULL DATASETS ANALYSIS}

Figure 16 reports the detrended light curves obtained using the whole data sets. As stated in Section 2.3, the systematics originally present in the raw light curves are greatly reduced (see Figure 1), but there are still visible trends at all phases of the transits. Quantitative measurements of these trends, based on the correlations with the pixel-phase, have been discussed in Section 3.1, together with the trends obtained by rejecting the first 450 data points before processing. Also, the standard deviations of residuals between the light curves and the transit models are larger for the cases with no preliminary data rejection (but for Obs $1 \mathrm{~b}$ the difference is not significant). Removing the first 450 data points after ICA processing may reduce the scatter, but it does not improve the reliability of the light curves, because trends are present at all times.

Results extracted from these worse-quality light curves are less robust, but they are consistent with accepted results within $1 \sigma$. Also, error bars are largely underestimated if the uncertainties due to the detrending process are neglected. It may lead us to erroneously detect inter-epoch transit depth variations.

\section{APPENDIX C ALTERNATIVE TRANSIT MODEL-FITS}

\section{C.1 Free Limb Darkening}

We performed transit model-fits with one free limb darkening parameter (linear or quadratic) in addition to the other free parameters $\left(p, a_{0}\right.$, and $\left.i\right)$. The standard deviations of residuals between the detrended light curves and the transit models do not change, among the models obtained with Atlas, Phoenix, free linear, and free quadratic limb darkening coefficients. The parameter error bars are larger by factors in the range of 1-3 for the free quadratic case, and much larger for the free linear case. Best parameter estimates may be more affected by intercorrelations. The pure quadratic limb darkening is a better approximation of the real case, because in both Atlas and Phoenix models the quadratic coefficients are greater than the linear ones. Figure 17 reports the estimates for the quadratic limb darkening coefficients, $\gamma_{2}$.

Note that:

1. they are comparable (within $1 \sigma$ ) with the theoretical values;

2. error bars are larger than the differences between Atlas and Phoenix values;

3. error bars do not allow us to distinguish the values at 3.6 and $4.5 \mu \mathrm{m}$.

Interestingly, the best estimate of $\gamma_{2}$ for Obs $1 \mathrm{~b}$ is the most distant from the other values (but within $1 \sigma$ ), and the correspondent transit duration is now equal for Obs 1a and 1b. Although the value $\gamma_{2} \sim 0$ is not reliable, it is important to note that the measured transit duration, as defined in Equation (3), depends on the stellar intensity distribution and limb darkening model adopted, then:

1. stellar variability is a possible cause for observed TDVs;

2. TDVs measured from observations at different wavelengths must be taken carefully.

\section{C.2 Free Eccentricity}

We performed transit model-fits with free eccentricity, $e$, in addition to the other free parameters $\left(p, a_{0}\right.$, and $\left.i\right)$. Eccentricity does not have a great impact on the transit models: best estimates of the other parameters do not change significantly with respect to previous ones (with $e=0.16$ ), although best estimates for the eccentricity for different observations varies over a large range (0.08-0.22). Also, residuals between light curves and models are not affected.

\section{C.3 Free Argument of Periastron}

We performed transit model-fits with free argument of periastron, $\omega$, in addition to the other free parameters $\left(p, a_{0}\right.$, and $i)$. They do not constrain $\omega$ very well $\left(\sigma_{\omega} \sim 24-34\right): a_{0}$ and $i$ are strictly correlated with $\omega$, and their error bars are $\sim 3$ times larger than ones obtained with $\omega$ fixed. Also, the distributions of $\omega, a_{0}$, and $i$, are asymmetric, because best $\omega$ values are close to the edge of the range of admissible values. It is interesting to note that other parameters, such as $p, b$, and $T$, are not affected by $\omega$ degeneracies: their posterior distributions are indistinguishable from the ones obtained with $\omega$ fixed. 


\section{C.4 Free Phase-shift}

We performed transit model-fits with a free phase-shift in addition to other free parameters in order to investigate the effect of possible timing variations. Results are very similar for each fitting configuration, with no evidence of timing variation below $\sim 30$ s, as stated in Section 3.3. The free phase-shift does not affect other parameter estimates, except in the cases with free argument of periastron $(\omega)$ : timing variations and orbit precession are highly correlated.

\section{C.5 Fitting $p, a_{0}$, and $i$ with Components' Coefficients}

We experimented with an alternative method to estimate the transit parameters and the coefficients of the independent components simultaneously, by modeling the raw light curves as linear combinations of the components plus a transit model. In this way, we can investigate possible correlations between transit parameters and mixing coefficients, and test the stability of an ICA model over the whole observation. If results were significantly different than the ones obtained by estimating the mixing coefficients on the out-of-transit only, it would indicate that something different has happened during the transit, either astrophysical or instrumental in nature. For the data sets analyzed in this paper, results are consistent with accepted values within $1 \sigma$. It is worth noting that partial error bars, $\sigma_{\mathrm{par}, 0}$, obtained from the MCMCs are similar to the ones obtained with $p, a_{0}$, and $i$ only free parameters (in some cases even smaller). This indicates that the main cause of uncertainty attributed to the detrending method is not given by the mixing coefficients, but by the intrinsic errors on the components extracted.

\section{REFERENCES}

Allard, F., \& Hauschildt, P. H. 1995, ApJ, 445, 433

Allard, F., Hauschildt, P. H., Alexander, D. R., Tamanai, A., \& Schweitzer, A. 2001, ApJ, 556, 357

Alonso, R., Barbieri, M., Rabus, M., et al. 2008, A\&A, 487, L5

Asplund, M., Grevesse, N., Sauval, A. J., \& Scott, P. 2009, A\&ARv, 47, 481

Ballard, S., Christiansen, J. L., Charbonneau, D., et al. 2010, ApJ, 716, 1047
Barriga, E. S., Pattichis, M., Ts'o, D., et al. 2011, Med. Image Anal., 15, 35 Beaulieu, J. P., Tinetti, G., Kipping, D. M., et al. 2011, ApJ, 731, 16 Butler, R. P., Vogt, S. S., Marcy, G. W., et al. 2004, ApJ, 617, 580 Cáceres, C., Ivanov, V. D., Minniti, D., et al. 2009, A\&A, 507, 481

Coughlin, J. L., Stringfellow, G. S., Becker, A. C., et al. 2008, ApJL, 689, L149

Deming, D., Harrington, J., Laughlin, G., et al. 2009, ApJL, 667, L199

Demory, B.-O., Gillon, M., Barman, T., et al. 2007, A\&A, 475, 1125

Fazio, G. G., Hora, J. L., Allen, L. E., et al. 2004, ApJS, 154, 10

Ford, E. B., Quinn, S. N., \& Veras, D. 2008, ApJ, 678, 1407

Gillon, M., Pont, F., Demory, B. O., et al. 2007, A\&A, 472, L13

Haario, H., Laine, M., Mira, A., \& Saksman, E. 2006, Stat. Comput., 16, 339

Howarth, I. D. 2011a, MNRAS, 413, 1515

Howarth, I. D. 2011b, MNRAS, 418, 1165

Hyvärinen, A., \& Oja, E. 2000, NN, 13, 411

Hyvärinen, A., Karhunen, J., \& Oja, E. 2001, Independent Component Analysis (New York: John Wiley \& Sons, Inc.)

Igual, J., Vergara, L., Camacho, A., \& Miralles, R. 2002, Neurocomputing, 50,419

Jordán, A., \& Bakos, G. Á 2008, ApJ, 685, 543

Knutson, H. A., Madhusudhan, N., Cowan, N. B., et al. 2011, ApJ, 735, 27

Knutson, H. A., Benneke, B., Deming, D., \& Homeier, D. 2014, Natur, 505,66

Koldovský, Z., Tichavský, P., \& Oja, E. 2006, ITNN, 17, 1265

Kurucz, R. L. 1970, Smithsonian Astrophysical Observatory Special Report 309

Lagarias, J. C., Reeds, J. A., Wright, M. H., \& Wright, P. E. 1998, SIAM Journal of Optimization, 9, 112

Mandel, K., \& Agol, E. 2002, ApJL, 580, L171

Maness, H. L., Marcy, G. W., Ford, E. B., et al. 2007, PASP, 119, 90

Morales-Caldéron, M., Stauffer, J. R., Davy Kirkpatrick, J., et al. 2006, ApJ, 653,1454

Morello, G. 2015, ApJ, submitted

Morello, G., Waldmann, I. P., Tinetti, G., et al. 2014, ApJ, 786, 22

Pál, A., \& Kocsis, B. 2008, MNRAS, 389, 191

Ribas, I., Font-Ribera, A., \& Beaulieu, J. P. 2008, ApJL, 677, L59

Pont, F., Gilliland, R. L., Knutson, H., Holman, M., \& Charbonneau, D. 2009, MNRAS, 393, L6

Stevenson, K. B., Harrington, J., Nymeyer, S., et al. 2010, Natur, 464, 1161

Stevenson, K. B., Harrington, J., Lust, N. B., et al. 2012, ApJ, 755, 9

Stone, J. V., Porrill, J., Porter, N. R., \& Wilkinson, I. D. 2002, NeuroImage, 15,407

Tichavský, P., Koldovský, Z., Yeredor, A., Gómez-Herrero, G., \& Doron, E. 2008, ITNN, 19, 421

Torres, G. 2009, ApJL, 671, L65

Waldmann, I. P. 2012, ApJ, 747, 12

Waldmann, I. P., Tinetti, G., Deroo, P., et al. 2013, ApJ, 766, 7

Yeredor, A. 2000, ISPL, 7, 197 